\title{
Zum Sprachstil in Wissensvermittlungsfernsehsendungen für Kinder im deutsch-tschechischen Vergleich Ausgewählte Aspekte mit Fokus auf die Arbeit mit Fachwörtern
}

\author{
Eva POLÁŠKOVÁ
}

\begin{abstract}
A German-Czech language style comparison in children's educational TV programmes. Selected aspects focusing on work with specialised terms

School is not the only institution that educates children and provides them with specialised information. Television also contributes substantially to education. The article deals with the question of how the authors of educational programmes for children handled the difficult task of educating children while capturing their attention at the same time. This issue is examined using the example of selected German and Czech television programmes for children with the topic of biology, and attention is paid especially to the choice of language when working with specialised terms and to the specific procedures of defining or explaining them. Also monitored is whether or not the strategy of introducing new specialised terms in the analysed children programmes is different in terms of quality from the strategies applied in popular science programmes for adults with comparable topics.
\end{abstract}

Keywords: children, television programme, education, mediation of knowledge, technical term, define, explain, biology

DOI: doi.org/10.15452/StudiaGermanistica.2021.28.0009

\section{Einleitung}

Es ist allgemein bekannt, dass sich nicht nur die Schule selbst an der Bildung beteiligt. Kinder gewinnen Informationen auch aus anderen Quellen, zu denen unter anderem das Fernsehen gehört. Dieses Medium wird aber primär nicht direkt mit Wissensvermittlung verbunden, vielmehr setzen sich Kinder vor den Fernseher, um unterhalten zu werden. Das bringt die Autoren/Autorinnen der Wissensvermittlungsfernsehsendungen für Kinder in keine leichte Situation. Einerseits setzen sie sich zum Ziel, Kinder zu belehren, andererseits wollen sie Kinder nicht enttäuschen oder langweilen.

Solche Fernsendungen zeichnen sich deswegen durch einen spezifischen Charakter und einen spezifischen Sprachstil aus, der analysebedürftig ist. Die folgende Analyse strebt an, diesen Stil am Beispiel der ausgewählten Wissensvermittlungsfernsehsendungen für Kinder im deutschen und 
tschechischen Sprachraum unter die Lupe zu nehmen und zu vergleichen. Weil es sich um ein breites Thema handelt, das viele Subthemen umfasst, wird die Aufmerksamkeit vor allem auf ausgewählte Aspekte der Arbeit mit den Fachwörtern gerichtet, die für eine Wissensvermittlung typisch sind. Es werden Passagen analysiert, die Fachwörter auf verschiedene Art und Weise behandeln.

Konkret wurden bei der Analyse folgende Fragen gestellt:

- Wie werden Fachwörter neu eingeführt und im Kontext präsentiert? Wie wird den Kindern beigebracht, dass es sich bei dem folgenden Wort um ein Fachwort handelt? Werden sie bei den Beschreibungen nur nebenbei erwähnt oder wird darauf besonders hingewiesen? Wie werden Fachwörter erklärt/definiert?

- Wie wird die Gliederung des Textes mit Fachwörtern vorgenommen?

- Können Unterschiede zwischen den tschechischen und deutschen Erklärungen in den analysierten bildenden Fernsehsendungen für Kinder beobachtet werden?

- Auf welche Art und Weise kooperieren die verbalen Elemente einerseits und die nonverbalen und paraverbalen Elemente andererseits bei den Erklärungen/Definitionen ${ }^{1}$ von Fachwörtern?

- Weisen Wissensvermittlungsfernsehsendungen für Kinder Spezifika im Vergleich zu den vergleichbaren Fernsehsendungen für Erwachsene auf?

\section{Analysiertes Korpus}

Zur Analyse wurden vergleichbare Fernsehsendungen im deutschen und tschechischen Fernsehen ausgesucht, wobei die Vergleichbarkeit nach folgenden Kriterien bestimmt wurde:

- Thema der Sendung,

- vorausgesetztes Alter der Kinderzielgruppe,

- Typ der Sendung.

Es wurden vier Serien für Kinder analysiert, die in den Kanälen für Kinder und Jugendliche veröffentlicht werden und auch online zugänglich sind (der Kinderkanal KiKA von den deutschen Fernsehsendern ARD und ZDF [URL 1], der Kinderkanal Déčko vom tschechischen Fernsehsender Česká televize [Tschechisches Fernsehen] [URL 2]).

Konkret wurden folgende Fernsehendungen ausgewählt und der Analyse unterzogen:

Von KiKA:

- ,Neuneinhalb: Lexikon-Videos‘ (auch von dem deutschen Fernsehsender WDR): 14 Teile zu einem biologischen bzw. mit Natur zusammenhängenden Thema (URL 3), ${ }^{2}$

- ,Tierbabys mit der Ente': 20 Teile (URL 4). ${ }^{3}$

Von Déčko:

- ,Živý svět: Kvítí‘ [Lebendige Welt: Blumen]: 10 Teile (URL 5), ${ }^{4}$

- „Ptačí rodinky: To nejzajímavější z ptačího světa“ [Vogelfamilien: Das Interessanteste aus der Vogelwelt]: 13 Teile (URL 6). ${ }^{5}$

In diesem Artikel wird die Bezeichnung „Definition“ im breiteren Sinne des Wortes verstanden, d. h. als jede Erklärung oder Charakteristik, die den Rezipienten/Rezipientinnen das Fachwort näher erläutern sollte. In Anbetracht der analysierten Textsorte können keine offiziellen Definitionen von Termini wie in Fachwörterbüchern erwartet werden (zu Typen der Definitionen vgl. Arntz/Picht/Schmitz 2014).

Weiter verkürzt ,Lexikon‘. Die Anzahl der angebotenen Videos ändert sich im Laufe der Zeit.

Weiter verkürzt ,Tierbabys‘.

Weiter verkürzt ,Kvítí‘.

Weiter verkürzt ,Ptačí rodinky‘. 
Alle Fernsehsendungen oder Teile davon betreffen verschiedene Aspekte der Natur, es lässt sich voraussetzen, dass sie überwiegend bei Kindern der Primarstufe ${ }^{6}$ Interesse wecken, und es handelt sich jeweils um eine lexikonartige Serie (jeweils die ersteren) und eine beschreibende bzw. erzählende Serie (jeweils die letzteren).

Damit Spezifika der Fernsehsendungen für Kinder besser hervorgehen können, wurden eine tschechische und eine deutsche Fernsehsendung über Natur für Erwachsene in die Analyse einbezogen. Diese Sendungen sind nicht direktes Objekt der Untersuchung, sondern dienen nur dem Vergleich als Bezugsobjekt. Konkret wurden folgende Fernsehsendungen ausgewählt:

- ,Kielings wilde Welt‘ (ZDF, Terra X; URL 12), ${ }^{7}$

- ,My, chytř́i primáti“/[Wir klugen Primaten] (ČT, URL 16). ${ }^{8}$

Die tschechische Serie beinhaltet drei Teile (URL 17, 18, 19), die deutsche Sendung besteht aus mehreren Serienteilen. Wegen der Vergleichbarkeit wurden adäquat zu der dreiteiligen tschechischen Sendung nur drei möglichst vergleichbare Serienteile ausgewählt, in denen unter anderem Affen besprochen werden (URL 13, 14, 15).

\section{Methode der Untersuchung und theoretische Ausgangsbasis}

Für die Beantwortung der gestellten Fragen wurde die Methode der qualitativen Analyse ausgewählt, weil primär nicht die Anzahl, sondern die verschiedenen Typen der Erklärungen und Definitionen und deren potenzielle Wirkungen und Effekte bei den Rezipienten/Rezipientinnen hinterfragt werden. ${ }^{9}$ Das wird an ausgewählten Beispielen gezeigt und interpretiert.

$6 \quad$ Die Einschätzung des Alters von Kindern, für die die analysierten Fernsehsendungen bestimmt sind, wurde von der Autorin des Artikels nach der verwendeten Sprache und dem ganzen Konzept jeder Fernsehsendung und Andeutungen in Beschreibungen dieser Sendungen im Internet vorgenommen, weil eine genaue Angabe des empfohlenen Alters von den Fernsehsendern selbst direkt bei den Fernsehsendungen nicht beobachtet wurde. Meistens wird nur über „Kinder“ gesprochen, und zwar auch auf Websites oder in Dokumenten, die diese Sendungen oder deren übergeordnete Quelle beschreiben (vgl. URL 7, 8, 9, 10).

Lediglich wurde bei der ursprünglichen Serie, von der die Serie ,Tierbabys mit der Ente ' wohl übernommen wurde (,Andy‘s Baby Animals‘ von dem Rundfunk- und Fernsehsender BBC), folgende Beschreibung gefunden: „Preschool series exploring how baby animals learn skills such as climbing, swimming and getting around.“(URL 11). Es ist jedoch fraglich, wie relevant diese Information ist, wenn man bedenkt, dass es bei der Transformation aus der englischen in die deutsche Version logischerweise zu Übersetzungen (also Änderung der Sprache) und zu weiteren Änderungen gekommen ist, worauf einige in Youtube veröffentlichte englische Videos von dieser Reihe hindeuten (vgl. URL 30).

Die Einschätzung wird auch durch die Tatsache verkompliziert, dass die Kanäle, die diese Sendungen anbieten, verschiedene Altersgruppen der Kinder ansprechen wollen, sodass sich das empfohlene Alter durch Analogie mit anderen Sendungen auch nicht ableiten lässt. Des Weiteren ist zu beachten, dass sich Kinder individuell kognitiv entwickeln, eigene Präferenzen des Sprachstils (z. B. der gelesenen oder gehörten Texte) aufweisen und sich für unterschiedliche Themenbereiche interessieren. Deswegen wird hier die vorsichtige Annahme präsentiert, den Rezipientenkreis der analysierten Sendungen in das Alter von 5 bis ca. 10 Jahre zu situieren. Es wird aber nicht ausgeschlossen, dass auch jüngere oder ältere Kinder sich dafür interessieren.

Ein Indiz für Bestimmung, welches Alter gemeint ist, ist in der tschechischen Sendung ,Ptačí rodinky das Wort „Ferien“. Es lässt sich vermuten, dass schon die Kinder im Schulalter gemeint werden, denn für die Kinder im Kindergarten sind Ferien nicht ganz relevant (URL 6, Serienteil: ,Čápovic zlatíčka“ [Goldkinder der Störche]). Auch die Konzeptionen der Fernsehsendungen können bei der Einschätzung nicht ganz weiterhelfen, denn überwiegend sind sie konkret und anschaulich verfasst, was auf eine präoperationale Phase der kognitiven Entwicklung/die frühe Kindheit (insgesamt 2-6/7 Jahre, Berk 2020:24, 314, in Anlehnung an Piaget) hindeuten könnte. An einigen Stellen wird dagegen komplexeres/logisches Denken voraussetzt, das vor allem in der mittleren Kindheit ansetzt (insgesamt 6/7-10/11 Jahre, vgl. Berk 2020:444-473). Diese Annahme wird in zwei analysierten Sendungen darüber hinaus dadurch unterstützt, dass bei einigen Animationen eine Schrift verwendet wird, die Vorschulkinder nicht entschlüsseln könnten (siehe unten). Weiter verkürzt, Wilde Welt‘.

8 Weiter verkürzt ,Primáti`. Diese Sendung wurde von dem Rundfunk- und Fernsehsender BBC (,Monkeys Revealed`) übernommen.

9 Die quantitative Analyse könnte dann die Relevanz der exemplarisch exzerpierten Sprachmittel nach deren Frequenz bekräftigen (siehe Fazit). 
Die Auswahl des Verfahrens für die Analyse ist mit mehreren methodologischen Herausforderungen verbunden.

Die erste methodologische Herausforderung stellt die Gliederung der Analyse dar, weil verschiedene Texte verglichen werden und verschiedene Aspekte ins Spiel kommen - Alter des Publikums, Sprache der Sendung, Konzept der Sendung usw. Um sich auf die sprachliche Seite konzentrieren zu können und vor allem den Sprachstil vergleichen zu können, war es notwendig, die anderen Variablen zu reduzieren. Die Autorin des Artikels ließ sich durch verschiedene Theorien inspirieren, die im Rahmen mehrerer linguistischer Disziplinen entstanden und auf einen Vergleich der sich minimal unterscheidenden Elemente abzielten. ${ }^{10}$ Ausgehend von diesen Methoden wurde bei der Analyse so vorgegangen, dass für die Analyse nach am meisten ähnlichen Passagen gesucht wurde. Die Ähnlichkeiten betrafen mehrere Parameter - die Passagen mussten nicht unbedingt inhaltliche Ähnlichkeiten aufweisen, sondern auch formale, organisatorische oder theoretische Aspekte. ${ }^{11}$ So konnte die Aufmerksamkeit auf die Unterschiede in den einzelnen Hauptparameter gerichtet werden, z. B. im Zielpublikum und in der Sprache. Dadurch kann erreicht werden, dass die Unterschiede vor den Hintergrund treten. Der Nachteil von dieser Darstellung besteht dagegen darin, dass die Beispiele nicht immer aus allen analysierten Fernsehsendungen stammen bzw. wegen des begrenzten Umfangs stammen können, sodass die Vergleiche nicht immer symmetrisch sind. ${ }^{12}$ Bei der Analyse wurden vor allem folgende Dimensionen fokussiert:

- wie die sprachliche Erklärung selbst gestaltet wird, z. B. ob zuerst das Denotat beschrieben/definiert und erst danach das Fachwort angegeben wird (kataphorische Fachworterklärung) oder umgekehrt (anaphorische Fachworterklärung) (Definitionstypen vgl. bei Niederhauser 1999:146), ${ }^{13}$

- ob und wie die Definition eingeleitet wird, d. h. ob und wie auf das Fachwort hingewiesen wird (vgl. implizite und explizite Einführung der Termini von Hahn 1983:94-97), ${ }^{14}$

- wie der Kontext und die Textgestaltung aussehen, d. h., textlinguistische Strukturen/Aspekte wurden involviert, z. B. Kohärenz und Kohäsion (vgl. Wolf 2008), oder syntaktische und stilistische Besonderheiten (Eroms 2008), die im Zusammenhang mit dem Effekt solcher Textgestaltungen auf den Rezipienten erörtert wurden (vgl. Problematik der Textverständlichkeit bei Göpferich 2001).

Die zweite methodologische Herausforderung ist mit der Interdisziplinarität dieser Problematik verbunden, d. h., um die Fragen beantworten zu können, musste sich die Autorin des Artikels an mehrere theoretische Konzepte und Bereiche anlehnen. Zu diesen gehören neben den oben genannten die Sprache der Massenmedien (vgl. Burger/Luginbühl 2014), vermittelnde Fachsprache bzw. populärwissenschaftliche Vermittlungsfachsprache (vgl. Niederhauser 1999) ${ }_{15}^{15}$ Sprache der Kinder vs. der Erwachsenen (transitorische Soziolekte) (vgl. Löffler 2010:117-120), Entwicklungspsychologie (siehe oben), Sprache der Biologie (vgl. Fässler 1998), gesprochene Sprache (vgl. Schwitalla 2012)

10 Z. B. vgl. die Theorie der Minimalpaare im Rahmen der Phonetik (Tworek 2019), minimale semantische Differenz bei partiellen Synonymen im Rahmen der Lexikologie (Römer 2019:136), Methode des Vergleichs der sich minimal unterscheidenden Elemente im Rahmen des Textes (Langer/Schulz von Thun/Tausch 1974, Göpferich 2001) und im Rahmen der Grammatik (Breindl/Wassner 2006) usw.

11 Z. B. Typ der Definition, einleitende Ausdrücke, Strategien bei der Erklärung, Gliederung des Textes mit Fachwörtern usw.

12 Es wurde angestrebt, Beispiele aus den Sendungen für Kinder und für Erwachsene in beiden analysierten Sprachen gegenüberzustellen, zugleich aber unnötige Redundanzen zu vermeiden.

13 Die weiteren Klassifikationen der Definitionsarten, z. B. nach Arntz/Picht/Schmitz (2014:64-65) und Roelcke (2010:60-68) werden nur bei eindeutigen Beispielen genannt.

14 Das spielt vor allem bei dem Kinderpublikum eine wichtige Rolle, damit die Kinder wissen, wie der Status des Fachwortes ist und was ihre Aufmerksamkeit verdient.

15 Vgl. dazu Theorie der Fachsprachen bei Hoffmann (1998); Fraas (1998); Roelcke (2010). Zur tschechischen Fachsprache bzw. zum tschechischen Fachstil vgl. Čmejrková/Daneš/Světlá (1999); Kozubíková Šandová (2019). 
usw. Des Weiteren muss der multimodale Charakter des analysierten Materials in Betracht gezogen werden (vgl. Siever 2015, Bucher 2017).

Bei multimodalen Texten ist es problematisch, sprachliche von den nicht-sprachlichen Elementen völlig zu teilen und sich nur dem ,reinen“ Sprachstil zu widmen, weil nonverbale Elemente eine wichtige Rolle spielen. Man kann einerseits diesen Bestandteil der analysierten Fernsehsendungen nicht übergehen, andererseits übergreift eine vollständige, komplexe Analyse der nonverbalen Mittel den Umfang dieses Artikels. Bei der folgenden Analyse der Arbeit mit den Fachwörtern wird deswegen von sprachlich-kommunikativen Aspekten der deutschen und tschechischen untersuchten Sendungen ausgegangen, um Unterschiede im Sprachstil festzustellen, und das visuelle Begleitmaterial und die Verbundenheit mehrerer Zeichensysteme und deren Funktionen und Beteiligung an der Wissensvermittlung wurde vor allem analysiert, wo es für die Textpassage und die Wissensvermittlung bzw. das Verständnis relevant ist und der Sprachstil durch nonverbale Mittel besonders beeinflusst ist. Die Autorin orientierte sich vor allem an der Gliederung der Bilder nach Weidenmann (1994:12-25), Stöckl (2004:250) und Burger/Luginbühl (2014:407-443). ${ }^{16}$

Die dritte methodologische Herausforderung bezieht sich auf die Darstellung der herausgefundenen Unterschiede und Spezifika. Da kohärente Passagen mit einem gewissen Kontext, nicht einzelne Wörter, analysiert wurden, konnten sich durch die oben beschriebene Methode nicht nur Unterschiede in einer Erscheinung oder einem Zeichen herauskristallisieren. In ein und demselben Satz oder einer und derselben Passage ließen sich häufig mehrere analysierte Aspekte und sprachliche Merkmale demonstrieren. Um Wiederholungen zu vermeiden, werden ausgewählte Passagen nur einmal bei einer Hauptproblematik komplex samt allen relevanten Phänomenen analysiert und es wird darauf bei anderen Subthemen gegebenenfalls nur noch wieder hingewiesen.

Auf die vierte methodologische Herausforderung wird bei allen Werken eingegangen, die sich mit Fachwörtern beschäftigen. Es stellt sich nämlich die Frage am Anfang heraus, welche Wörter als Fachwörter zu betrachten sind (vgl. von Hahn 1983:83-106, Arntz/Picht/Schmitz 2014). Da diese Frage bei der Fragestellung in diesem Artikel keine primäre Rolle spielt, ist die Autorin intuitiv vorgegangen und hat Fachwörter in die Analyse einbezogen, die Fachbegriffe aus dem Bereich der Natur oder den zusammenhängenden Bereichen benennen und abgrenzen und nicht ganz zu den basalen Kenntnissen der Kinder bzw. der Erwachsenen zählen, sodass die Wörter auch in der Alltagssprache geläufig verwendet würden. Des Weiteren ist ein „Erkennungszeichen“ für die zu analysierenden Fachwörter gerade die Tatsache, was in der Fernsehsendung selbst näher erklärt, hervorgehoben und/oder worauf explizit hingewiesen wird (siehe oben).$^{17}$

\section{Konzepte der Fernsehsendungen im Vergleich}

Bevor die Ergebnisse der Sprachanalyse gezeigt werden können, sollten allgemeine Konzepte der Fernsehsendungen vorgestellt und verglichen werden, d. h. inhaltliche, kontextuelle und extraverbale

16 Typen der Bilder nach Weidenmann (1994:12-25): Abbilder, logische Bilder, Kombination von logischen Bildern mit Abbildern, schematische Bilder, Analogiebilder. Vgl. semiotische Aspekte nach Burger/Luginbühl (2014:413-417): Ikon, Index, Symbol.

Stöckl (2004:250, in Anlehnung an Kalverkämper 1993) unterscheidet mehrere Typen der Bild-Sprache-Beziehungen, z. B. redundante Beziehung, komplementäre Beziehung, dominante Beziehung. Vgl. redundante Beziehung, komplementäre Beziehung auch bei Burger/Luginbühl (2014:425-438) zusammen mit rhetorischen Beziehungen (Metonymie, Metapher) und weiteren Beziehungen. Vgl. Terminologie bei Niederhauser (1999:190, in Anlehnung an Kalverkämper 1993): gleichwertige, überwertige/textdominierende, unterwertige Bilder. Vgl. Funktionen des Bildes (Burger/ Luginbühl 2014:438-443).

Anmerkung: Stöckl betrachtet Bilder und Texte als eine Einheit, der Übersicht halber werden aber in diesem Artikel Texte und Bilder als getrennte, wenn auch zusammenhängende Objekte analysiert.

17 Die definierten Fachwörter werden geläufig als Termini bezeichnet (zur Problematik der Terminologiearbeit vgl. Arntz/ Picht/Schmitz 2014:27, 64-65). Weil zu den „Definitionen“ in diesem Artikel vor allem inoffizielle Definitionen/Erklärungen zählen, nicht die offiziellen Definitionen aus einem Fachwörterbuch, werden die untersuchten Wörter, die Fachinhalte bezeichnen, „Fachwörter“ und nicht Termini genannt. 
Aspekte, weil diese die Sprache der Sendung prägen oder beeinflussen können. Es wurde im ersten Schritt nach Unterschieden zwischen den analysierten Kinderfernsehsendungen gesucht, dazu wurden dann im zweiten Schritt Fernsehsendungen für Erwachsene herangezogen, um Spezifika der Kinderfernsehsendungen herauszufiltern.

\subsection{Kindersendungen}

Alle analysierten Fernsehsendungen betreffen Natur, sie unterscheiden sich jedoch darin, was akzentuiert und wie die Fernsehsendung organisiert wird.

Im Rahmen der lexikonartigen Serien für Kinder (,Lexikon', „Kvítí') werden verschiedene Begriffe kurz erklärt und von Aufnahmen aus der Natur und graphischen Visualisierungen begleitet. In ,Lexikon` erfahren die Kinder Grundinformationen über ausgewählte Natur-Erscheinungen, wobei diese nicht miteinander zusammenhängen und zusammen mit Videos zu anderen Themen, z. B. gesellschaftlichen, angeboten werden. Die Videos sind nicht nach Themen, sondern alphabetisch angeordnet. Beispielsweise findet man hier erklärende Videos zu ,Alexander Humboldt', ,Algorithmen', ,Allergien‘, ,Amputierten Fußball‘ über ,Bienen`, ,Drillinge‘, ,Eintagsküken` bis ,Vitamine‘, ,Vivaldi, Antonio Lucio“ und ,Wölfe‘. Die tschechische lexikonartige Serie ,Kvítí k konzentriert sich nur auf die Beschreibung und Charakteristik von Pflanzen, mit denen Kinder geläufig in Kontakt kommen, weil jeder - nach der Autorin dieser Sendung - diese kennen sollten und die Kinder sie wohl nicht einmal benennen können, z. B. Löwenzahn, Gänseblümchen, Klee usw. (URL 10)

Bei den beschreibenden Serien für Kinder werden realistische Aufnahmen/Videos aus der Natur präsentiert und kommentiert, wobei man sich bemüht, der Beschreibung auch Züge einer Geschichte $\mathrm{zu}$ verleihen. In der Sendung ,Tierbabys' befasst man sich mit Jungtieren verschiedener Tierarten und die Serienteile sind nach den Tätigkeiten gegliedert, die Jungtiere ausüben (z. B. Serienteile ,Erste Schritte', ,Klettern', ,Trinken'), oder danach, wo sie leben (Serienteil ,Zu heiß, zu kalt') oder danach, wen sie zum Leben brauchen (Serienteil ,Super Mamis'). In jeder Serie werden ca. drei verschiedene Orte/Tierarten vorgestellt. Die tschechische Sendung ,Ptačí rodinky“ ist ähnlich konzipiert, es geht auch um Jungtiere, aber nur von ausgewählten Vogelarten, und zwar Störche, Schwalben, Stare und Turmfalken. Die Serienteile sind chronologisch konzipiert, jeder Art sind drei Serienteile gewidmet und es wird die Zeitspanne von Zusammentreffen von Weibchen und Männchen über Schlüpfen bis zum Selbstständigwerden von Jungtieren dokumentiert.

$\mathrm{Zu}$ beachten ist auch der formale Rahmen aller analysierten Kindersendungen, d. h., wie die einzelnen Serienteile eingeleitet und abgeschlossen werden. Bei der Sendung „Tierbabys“ werden Kinder mit einer animierten sich bewegenden Ente $^{18}$ begleitet, die am Anfang gezeigt wird, und danach kommen Videos der realen Natur. Die Sendung ,Ptačí rodinky“ beginnt und endet auch mit einer Animation fliegender Vögel, wobei der Titel der Sendung und beteiligte Personen am Ende gezeigt werden. Es wirkt ein bisschen wie Einführung und Anschluss eines Fernseh-Märchens bzw. es erinnert an die Form des täglichen Abendmärchens im Fernsehen für tschechische Kinder (,Večerníček'/[Sandmännchen], vgl. URL 29).

Auch die lexikonartigen Serien haben immer eine spezifische Einleitung. Während am Anfang der deutschen Sendung „Lexikon“ immer eine Kennmelodie mit Titel des Programms „Neuneinhalb" kommt (es wirkt wie eine Werbung) und erst danach die Bezeichnung der präsentierten Erscheinung per Buchstaben schrittweise gezeigt/getippt wird (diese Schrift erinnert an die Schrift einer Schreibmaschine), ist am Anfang der tschechischen Sendung ,Kvítí 'immer die allgemeine Überschrift „Živý svět. Kvíti““/[Lebendige Welt: Blumen] zu sehen, indem eine Animation zeigt, wie die Blume vom Samen aufwächst. Dann werden Grundinformationen gesagt und erst danach kommt die Bezeichnung der Blume auch in handgeschriebener Schrift auf dem Bildschirm. Es erinnert ein bisschen an die Schreibweise, wie es Kinder in ihre Hefte in der Schule schreiben würden.

Die Länge der einzelnen Sendungen entspricht dem Alter der vorausgesetzten Rezipienten und deren Fähigkeit sich nur eine gewisse Zeit konzentrieren zu können. Die einzelnen Sendungen

18 D. h. Kategorie Abbilder (vgl. Weidenmann 1994:12-25) bzw. Ikonen (vgl. Burger/Luginbühl 2014:413-415). 
für Kinder dauern ca. 1 bis 3 Minuten (lexikonartige Serien) oder ca. 4 bis 10 Minuten (erzählende Serien).

\subsection{Erwachsenensendungen zum Vergleich}

Wenn man das Konzept der vergleichbaren Sendungen für Erwachsene analysiert, zeigen sich gewisse Unterschiede, wenn auch nicht große.

- Die Sendungen für Erwachsene sind komplexer, besprechen mehrere Subthemen (auch z. B. Schutz der Natur, Nationalparks, Ereignisse in der Geschichte usw. bei der deutschen Sendung). Mit dem Charakter ähneln sie am meisten der Kindersendung ,Tierbabys', d. h. sie werden überwiegend durch Aufnahmen aus der Natur gebildet.

- Die verglichenen Serienteile für Erwachsene sind länger, sie dauern über 40 Minuten, was damit zusammenhängt, dass die Erwachsene ihre Aufmerksamkeit länger halten können als Kinder.

- Während bei den Sendungen für Kinder vor allem Tiere auftreten und der Mensch meistens nicht eingreift, werden in den Sendungen für Erwachsene Aktivitäten der Menschen markanter präsentiert. Zum Beispiel führt man Gespräche mit Wissenschaftlern/Wissenschaftlerinnen oder die Menschen bewegen sich unter Tieren und helfen ihnen. Bei der deutschen Serie ,Wilde Welt' gibt es einerseits einen Kommentator, der die ganze Sendung sprachlich begleitet und die Aufnahmen erklärt, andererseits tritt der Moderator (Andreas Kieling) ab und $\mathrm{zu}$ in Videos auf, er ist direkt vor Ort und kommentiert den Kontakt mit den Tieren oder er interviewt Wissenschaftler/Wissenschaftlerinnen, an deren wissenschaftlicher Expedition er sich beteiligt. Die Namen und Titel der Wissenschaftler/Wissenschaftlerinnen werden explizit in Titeln genannt, was ihre Gelehrsamkeit nachweisen soll. Dieses Konzept der Sendung ist interaktiv und intradiegetisch (Burger/Luginbühl 2014:421).

- In der tschechischen Sendung gibt es keinen solchen Moderator vor Ort wie Andreas Kieling, in ,Primáti“ werden nur Videoaufnahmen mit Tieren gezeigt, der Kommentator kommt nicht darin vor (extradiegetisches Konzept, Burger/Luginbühl 2014:421). Vereinzelt werden jedoch Wissenschaftler/Wissenschaftlerinnen in der Natur gezeigt und ihre Arbeit wird kommentiert, z. B. wie zwei Zoologinnen einen Versuch in der Natur in Südafrikanischen Republik anlegen (Serienteil ,Důležitost rodiny“/[Wichtigkeit der Familie], URL 18) oder wie Menschen mit verwaisten Affen Leben in der wilden Natur üben (Serienteil ,Dar inteligence“/[Gabe der Intelligenz], URL 19). Ihre Tätigkeiten werden nur vom Kommentator thematisiert, sie werden nicht interviewt. ${ }^{19}$

- Bei der Analyse der Sprache treten auch inhaltliche Unterschiede in den Vordergrund. Obwohl sie nicht im Zentrum der Aufmerksamkeit stehen, kann an dieser Stelle mindestens auf einen Unterschied hingewiesen werden, und zwar, dass Sendungen für Erwachsene unter anderem grausame Seiten des Lebens zeigen, z. B. Jagd der Tiere, indem sie andere Tiere fressen (die tschechische Sendung ,Primáti'). In einigen analysierten Kindersendungen wird das zwar auch erwähnt, aber nicht explizit gezeigt, vielmehr beinhalten die Videos die Szenen, wie es einem Tier gelungen ist, einem Prädator zu entkommen. In der deutschen Sendung stellt eine gewisse Grausamkeit eine Verletzung des Affen dar, die beschrieben wurde. Die Kindersendungen weisen dagegen eine Tendenz auf, die positiven Seiten des Lebens bzw. das Leben als Spiel zu skizzieren. ${ }^{20}$

19 Das kann sogar an einer gleichen Rettungsaktion konkretisiert werden: Wissenschaftler/Wissenschaftlerinnen und Pfleger/ Pflegerinnen zeigen in der deutschen Sendung (URL 13) den Affen, wie sie Pflanzen/Ranken mit Dornen essen sollen, wobei die Tätigkeit direkt von dem Wissenschaftler im Video beleuchtet wird. In der tschechischen Sendung wird alles nur vom Kommentator kommentiert, die Menschen im Video erklären es den Rezipienten/Rezipientinnen nicht (URL 19).

20 Es handelt sich hier nur um eine Vorforschung, für genauere Ergebnisse wäre es notwendig, eine Inhaltsanalyse durchzuführen. 
- Vereinzelt werden in den deutschen Sendungen für Erwachsene neben Videos aus der realen Natur auch skizzierte Abbilder/Animationen eingesetzt, die z. B. das Verschwinden des Regenwaldes andeuten oder die Erkennung der Gorillas an deren Nasenfalter erklären, indem die Nasen vergrößert und durch eine Skizze abgebildet werden. Es werden selten Animationen genutzt, z. B. eines ausgestorbenen Vogels (Elephantenvogel, URL 13). Mithilfe eines animierten Modells wird die Anatomie des Affen beschrieben oder es werden alte Photographien von Wissenschaftlern/Wissenschaftlerinnen aus der Geschichte gezeigt. ${ }^{21}$ Die Verwendung von Animationen neben den realen Aufnahmen haben die deutschen Fernsehsendungen für Erwachsene mit den Kindersendungen ,Kvítí " und ,Lexikon“ gemeinsam, dagegen konzentrieren sich die Kindersendungen ,Tierbabys“ und ,Ptačí rodinky“ überwiegend auf die realen Aufnahmen aus der Natur.

- Leichte Unterschiede bestehen auch in den paraverbalen Mitteln, die die ganze Wirkung der Sendungen untermalen bzw. unterstreichen, indem wichtige oder interessante Tatsachen mit einer modifizierten Intonation gelesen werden. Die Stimme des Kommentators in den Sendungen für Kinder weist Emotionen auf und löst sie aus. Die tschechische Sendung ,Kvítí‘ wird sogar vom Kind (Mädchen) selbst gelesen, sodass sich die Kinder mit der Rolle der Wissenschaftler/Wissenschaftlerinnen identifizieren können. Die Stimme des Kommentators in den Sendungen für Erwachsene ist dagegen dunkel und ernst. Es wird wohl angestrebt, dass die durch die Stimme mitgeteilten Informationen seriös wirken. Diese prinzipielle Gliederung gilt aber nicht hundertprozentig, denn die lexikonartige deutsche Serie ,Lexikon' für Kinder unterscheidet sich paraverbal nicht sehr von den Sendungen für Erwachsene. Und umgekehrt hört der/die erwachsene Rezipient/Rezipientin vom Kommentator ab und zu eine Stimme, die Emotionen verrät. Vor allem der im Video der deutschen Sendung auftretende Moderator Andreas Kieling moduliert bei der Mitteilung seine Stimme, wenn er etwas hervorheben oder Begeisterung auslösen will.

\section{Eingeführte Fachwörter und deren Erklärungen im Vergleich}

In allen analysierten Sendungen kommen Fachwörter aus dem biologischen Bereich und aus anderen, mit Biologie zusammenhängenden Gebieten (z. B. Naturschutzkunde, Chemie, Medizin usw.) vor. Sowohl in den deutschen als auch in den tschechischen Sendungen für Kinder und für Erwachsene wurden verschiedene Strategien beobachtet, wie mit Fachwörtern umgegangen wird.

Im Folgenden werden einige Vergleiche der ausgewählten Passagen aus analysierten Sendungen geboten, die bestimmte Parallelen aufweisen, damit im Endeffekt die Unterschiede zwischen den verglichenen Fernsehsendungen bzw. deren Gemeinsamkeiten in ausgewählten Aspekten hervortreten (vgl. Kapitel 3 zur Methodologie). ${ }^{22}$ Entweder werden Behandlungen und Kontexte gleichklingender Fachwörter in unterschiedlichen Fernsehsendungen (Unterkapitel 5.1.) oder verschiedene Formen der Definitionen und ihrer einleitenden Ausdrücke verglichen (Unterkapitel 5.2.). Dabei werden Vergleiche mit einer tragenden Bedeutung detailliert analysiert und ergänzende Aspekte kürzer beigefügt. Gesammelte Beispiele werden tabellarisch präsentiert. ${ }^{23}$

21 Bei der tschechischen Sendung für Erwachsene ist noch die gelegentliche Verwendung der Infrarotkamera interessant.

22 Bei allen präsentierten Beispielen handelt es sich um Transkriptionen der mündlichen Kommentare bei den analysierten Fernsehsendungen, die die Autorin des Artikels selbst vorgenommen hat (die schriftlichen Vorlagen der Kommentare hatte sie nicht zur Verfügung).

23 In den analysierten Fernsehsendungen kommen auch Fachwörter vor, die nicht definiert/erklärt werden. Man setzt wahrscheinlich voraus, dass sie bekannt sind oder dass die Bedeutung vom Kontext und vom visuellen Material abgeleitet wird. Diese Fachwörter werden in der Analyse nicht berücksichtigt (Beispiel: Dann beginnt das Herz kräftiger und schneller zu schlagen. Blutdruck und Puls steigen an. URL 25, ein Video-Lexikon-Beitrag für Kinder zum Fachwort ,Koffein'; die fettgedruckten Fachwörter werden nicht erklärt, die begleitenden Bilder zeigen nur ein Abbild von einer Frau mit Detail auf ihr pulsierendes Herz und daneben ein Kardiogramm mit den steigenden Kurven, es wird aber nicht erklärt, worin die Werte des Blutdrucks und des Pulses bestehen und wie sie errechnet werden.) 


\subsection{Arbeit mit gleichklingenden Fachwörtern im Vergleich}

Obwohl Themen der einzelnen analysierten Sendungen nicht ganz gleich waren, ist es gelungen, gleichklingende Fachwörter in einigen Fernsehsendungen zu finden. Zu diesen Wörtern gehören die Fachwörter Nektar oder Alpha-Männchen.

\subsubsection{Fachwort Nektar/nektar}

Das Fachwort Nektar kommt in der deutschen Fernsehsendung für Kinder ,Lexikon “ (Serienteil über Bienen, siehe Beispiel Nr. 1) und in der tschechischen Sendung für Kinder ,Kvítí (Serienteil über Klee, Beispiel Nr. 2) vor, wie der Tabelle 1 zu entnehmen ist. Dieser Vergleich ermöglicht Unterschiede zwischen den analysierten deutschen und tschechischen Fernsehsendungen für Kinder im Konzept der Erklärung zu verzeichnen.

\begin{tabular}{|c|c|}
\hline $\begin{array}{l}\text { K / D } \\
\text {,Lexikon“ }\end{array}$ & $\begin{array}{l}\text { Beispiel } 1 \\
\text { Bienen. Bienen erfüllen eine der wichtigsten Aufgaben in unserer Natur. Sie bestäuben } \\
\text { Blütenpflanzen, wie zum Beispiel Apfelbäume. Auf der Suche nach Futter fliegen die Tiere von } \\
\text { Blüte zu Blüte. Mit ihrem Rüssel saugen sie den süßen Nektar einer Blüte auf. Dabei bleibt } \\
\text { an ihrem haarigen Körper Blütenstaub - auch Pollen genannt - hängen. Fliegen sie nun zur } \\
\text { nächsten Blüte, um dort Nektar aufzusaugen, bleiben meist ein paar Pollen auf der Narbe } \\
\text { dieser Blüte kleben. Durch diese Bestäubung kann eine Frucht wachsen. [...] } \\
\text { URL } 20\end{array}$ \\
\hline $\begin{array}{l}\text { K / T } \\
\text {,Kvítí }\end{array}$ & $\begin{array}{l}\text { Beispiel } 2 \\
\text { [...] Květy jsou nejčastěji světle rǔžové až tmavě fialové, u jiných druhủ i billé. Skoro nevoní. } \\
\text { Jsou nektarodárné, to znamená, že produkuji sladký nektar. Pro včely však bývá nedostupný, } \\
\text { nedosáhnou na něj. Jednotlivé květy jsou hluboké, úzké trychtýřky, proto je múže opylovat jen } \\
\text { hmyz s dlouhým sosákem, především čmeláci a motýli. [...] } \\
\text { [Die Blüten sind am öftesten hellrosa bis dunkelviolett, bei anderen Arten auch weiß. } \\
\text { Sie riechen fast nicht. Sie sind Nektar gebend, das bedeutet, dass sie einen süßen Nektar } \\
\text { produzieren. Für Bienen ist er jedoch unzugänglich, sie erreichen ihn nicht. Einzelne Blüten } \\
\text { sind tiefe, schmale Trichterchen, deswegen kann nur das Insekt mit einem langen Rüssel sie } \\
\text { bestäuben, vor allem Hummeln und Schmetterlinge.] } \\
\text { URL } 5 \text { (Serienteil: Jetel/[Klee]) }\end{array}$ \\
\hline
\end{tabular}

Tab. 1: Arbeit mit gleichklingenden Fachwörtern im Vergleich (Fachwort Nektar/nektar)

Anmerkung: $\mathrm{K}=$ Fernsehsendung für Kinder, $\mathrm{E}=$ Fernsehsendung für Erwachsene, $\mathrm{D}$ = deutsche Fernsehsendung, $\mathrm{T}=$ tschechische Fernsehsendung

Für beide Sendungen gilt, dass das Fachwort Nektar vielmehr bei der Charakteristik anderer Erscheinungen verwendet wird, sodass dessen Bedeutung nur indirekt charakterisiert wird bzw. von dem Text und dem visuellen Begleitmaterial abgeleitet werden muss. ${ }^{24}$ In beiden Fällen kann nur der Kontext den Kindern helfen, das Wort richtig zu verstehen. Anstatt es den Kindern sehr ausführlich zu erklären, haben die Autoren/Autorinnen in beiden Fällen die Strategie präferiert, das Denotat durch ein Attribut zu erläutern, da das Adjektiv süß/sladký/[süß] sehr aussagend ist und die ganze komplizierte Charakteristik ersetzt, ${ }^{25}$ weil sie eigentlich nicht notwendig ist. Relevant ist nur, dass diese Sache die Bienen/das Insekt sehr anlockt.

Auch so ist es aber nicht sicher, ob die (vor allem jüngeren) Kinder die Bedeutung richtig ableiten, es kann also passieren, dass sie dem Wort entweder keine konkrete oder nur eine vage

24 Bei diesem Vergleich ging es vor allem um eine Analyse der vergleichbaren Passagen und Definitionen weiterer Fachwörter. Dass das Fachwort Nektar durch keine eindeutige Definition erklärt wird, wurde nicht beachtet.

25 Es erinnert an die Definitionsart „Angaben zur materiellen Beschaffenheit“ (Arntz/Picht/Schmitz 2014:64-65) bzw. ,explikative Definition“ bei Roelcke (2010:64-65). 
Bedeutung oder eine Bedeutung zuschreiben, die sie aus anderen Kontexten kennen. ${ }^{26}$ Dadurch ist die Definition des Fachwortes nektarodárný/[Nektar gebend] in der tschechischen Sendung ,Kvítí fraglich, denn man verwendet für die Erklärung dasselbe Fachwort, das einen Bestandteil des zu definierenden Fachwortes bildet (nektar/[Nektar]). Auf die Bedeutung ist mithilfe des einleitenden Satzes to znamená/[das bedeutet] explizit hingewiesen.

In beiden Sendungen ist das Wort Nektar ein Bestandteil einer komplexeren Definition bzw. Erklärung, und zwar von einer Bestäubung (,Lexikon') bzw. einem Aufbau der Klee-Blüte (,Kvítí). In „Lexikon“ ist die Definition unauffällig, weil erst am Ende der Passage das Fachwort „Bestäubung“ angegeben ist (kataphorische Definition). Dass sich die vorangehende Passage eigentlich auf das Fachwort Bestäubung bezieht, darauf deutet das Demonstrativpronomen diese hin. ${ }^{27}$ Beigefügt wird noch die Charakteristik des Fachwortes Pollen, die bei der Beschreibung des Prozesses als eine andere Bezeichnung für Blütenstaub in einer Parenthese bezeichnet wird, ${ }^{28}$ was die Kohärenz des Textes (und dessen Verständnis) ein bisschen beeinträchtigen kann. Die Kohäsion der Passage wird dagegen durch Topik mit lexikalischer Inklusion ${ }^{29}$ (Unter- und Oberbegriff Bienen, Tiere) unterstützt und es ist auch stilistisch relevant, dass die gleichen Wörter nicht wiederholt werden.

Bei der tschechischen Sendung ist es bei der Beschreibung der Bestäubung, die der Charakteristik vom nektarodárný/[Nektar gebend] folgt, notwendig, den Körperbau des Tieres zu kennen, sonst könnte man meinen, dass die Tiere mit ihren Gliedern (analogisch zur Hand) nach dem Nektar reichen. Diese ein bisschen komplizierte Formulierung ist durch das Verb dosáhnout/[erreichen] verursacht, weil der Körperteil, den es betrifft, erst später angegeben ist, sodass man an eine üblichere Sache wie Glieder denken könnte. Es ist also eine gewisse Diskrepanz zu beobachten - einerseits ist die Sprache sehr leicht wie für Kinder im Vorschulalter oder an der Primarstufe/in der 1. Klasse, aber andererseits ist es notwendig, unterscheiden zu können, was nur als ein Witz, eine übertragene Bedeutung oder ein bildlicher Ausdruck gemeint ist, was verschwiegen ist und was der Realität entspricht. Da Kinder im Vorschulalter bzw. im Alter von 5-6 Jahren häufig ihre Fantasie einsetzen und ein bisschen durch magisches Denken beeinflusst werden können (vgl. Siegler et al. 2016:258), könnte zu einem Missverständnis kommen. Dazu kann ferner die Reihenfolge der Informationen beitragen. ${ }^{30}$

\section{Zusammenspiel des Verbalen und Bildlichen}

Das visuelle Material lässt sich bei der deutschen Fernsehsendung ${ }^{31}$ als komplementär bezeichnen, denn die Animation ergänzt geeignet den Kommentar und unterstützt die Vermittlung und das richtige Verständnis der gesagten Information. In der analysierten Passage aus der tschechischen Fernsehsendung nimmt das visuelle Material vielmehr eine redundante bzw. unterwertige Stellung ein, ${ }^{32}$

26 Zum Beispiel aus Lebensmittelgeschäften - vgl. eine von möglichen Definitionen nach dem Onlinewörterbuch des Dudenverlags: „Getränk aus zu Mus zerdrücktem, gezuckertem und mit Wasser [und Säure] verdünntem Fruchtfleisch (meist in Zusammensetzungen, z. B. Frucht-, Orangennektar“ (URL 26).

27 Das Wort „dieses“ zeigt, dass der vorher beschriebene Prozess die Bestäubung war, es ist auch aufgrund der Wortbildung ableitbar. Was aber die Befruchtung konkret bedeutet, ist nicht angegeben.

28 Vgl. Synonymendefinition nach Roelcke (2010:68).

29 Vgl. Wolf (2008:63).

30 Man muss wissen, dass Bienen Insekt sind, des Weiteren, was sosák/[Rüssel] bedeutet (das Wort ist nicht erklärt). Ohne dieses Wissen kann das Verb nedosáhnou irreführend sein. Hilfreich wäre eine andere Reihenfolge der Informationen: zuerst ein kompletter Aufbau der Blüte (lange Trichter), dann die Spezifizierung und Begründung, welches Insekt es bestäuben kann (dlouhý sosák/[langer Rüssel]), und erst danach die Angabe und Begründung, welches Insekt nicht geeignet ist. Dann kann vorauszusetzt werden, dass der Ausdruck nedosáhnou na něj/[sie erreichen ihn nicht] richtig verstanden wird. Möglich wäre weiterhin die Formulierung: Nedosáhnou na něj svým krátkým sosákem/[Sie erreichen ihn mithilfe ihres kurzen Rüssels nicht]. (Vgl. die Problematik der Textverständlichkeit, Göpferich 2001:130-132).

31 Es handelt sich um eine Animation/sich bewegende Skizzen (Kategorie: Abbild), die direkt den Flug einer Biene auf die Blüte und das Kleben und Übertragen der Pollen veranschaulicht. (Nicht einmal durch Bilder können aber alle Tatsachen veranschaulicht werden, z. B. gerade das konkrete Aussehen des Nektars).

32 Es wird eine Kombination gezeigt: zuerst wird Struktur der Blüte skizziert, dann ein Video mit realen Bienen und Pflanzen projiziert. 
denn die Hauptinformationen werden in sprachlichen Komponenten gesagt und das Bild illustriert nur das Gesagte, dem Verständnis hilft es nur teilweise. ${ }^{33}$

\subsubsection{Fachwort Alpha-Männchen/alfa samec}

Anhand des gleichklingenden Fachwortes Alpha-Männchen/alfa samec in der deutschen Sendung für Kinder ,Tierbabys` und der tschechischen Sendung für Erwachsene ,Primáti ‘ können prinzipielle Unterschiede zwischen Sendungen für unterschiedliche Alter verfolgt werden. ${ }^{34}$ Die Textausschnitte weisen sowohl Gemeinsamkeiten als auch Unterschiede auf, wie aus der Tabelle 2 hervorgeht.

\begin{tabular}{|c|c|}
\hline $\begin{array}{l}\text { K / D } \\
\text {,Tierbabys' }\end{array}$ & $\begin{array}{l}\text { Beispiel } 3 \\
\text { [...] Das ist der Chef. Er wird auch Alpha-Männchen genannt. Alle müssen ihm Respekt } \\
\text { zollen. [...] } \\
\text { URL } 21\end{array}$ \\
\hline $\begin{array}{l}\text { E / T } \\
\text {,Primáti` }\end{array}$ & 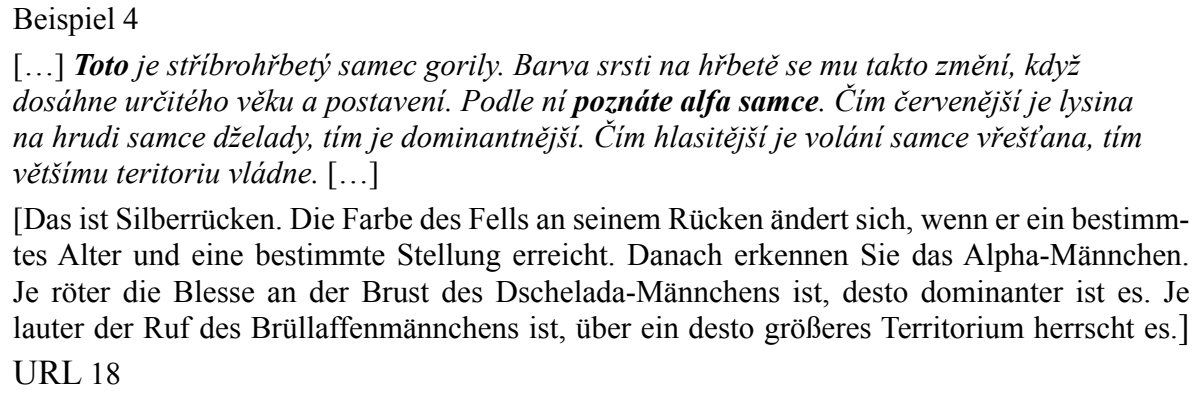 \\
\hline
\end{tabular}

Tab. 2: Arbeit mit gleichklingenden Fachwörtern im Vergleich (Fachwort Alpha-Männchen/alfa samec) Anmerkung: $\mathrm{K}=$ Fernsehsendung für Kinder, $\mathrm{E}=$ Fernsehsendung für Erwachsene, $\mathrm{D}=$ deutsche Fernsehsendung, $\mathrm{T}=$ tschechische Fernsehsendung

$\mathrm{Zu}$ den Unterschieden gehört der allgemeine Kontext, in den diese Problematik eingebaut wird. Während bei der deutschen Sendung für Kinder das umgangssprachliche Wort Chef als Ausgangspunkt dient und die Rolle des Männchens durch die Regeln in der Horde charakterisiert wird (was die anderen Tiere machen müssen), wird in der tschechischen Sendung für Erwachsene die Rolle der Farben bei der Charakteristik hervorgehoben, wobei die Regeln in der Gruppe implizit durch die Ausdrücke věk/[Alter], postaveni/[Stellung] einbezogen werden. Es werden zwei Sätze mit dem gleichen Anfangswort und parallelen Aufbau beigefügt (Anapher, Parallelismus), ${ }^{35}$ die die Problematik an konkreten Affenarten veranschaulichen und auch die Stellung durch die Wörter dominantnějššl [dominanter], vládne/[er herrst] explizit machen. ${ }^{36}$

Während das Fachwort in der deutschen Sendung für Kinder mithilfe eines passivischen Satzes und des Verbs nennen eingeführt wird, wird das Fachwort in der tschechischen Sendung für Erwachsene in einem Satz erwähnt, in dem die Rezipienten/Rezipientinnen direkt angesprochen werden (was man gerade umgekehrt hätte erwarten können).

33 Es hätte gerade das Abbild des Rüssels dem Verständnis sehr geholfen, es wird jedoch nur die Blüte und der lange Trichter animiert, nicht aber der lange und kurze Rüssel. Das Insekt wird nur im realen Video aus der Natur allgemein auf der Blüte gezeigt. Die Aufnahme ist nicht so detailliert, dass die Kinder den Rüssel genau identifizieren könnten (an dem Insekt sind z. B. auch Glieder oder Fühler sichtbar und das Insekt bewegt sich ziemlich schnell).

$34 \mathrm{Zu}$ einem vollkommenen Vergleich hätten diese Fachwörter noch in gleichsprachigen Fernsehsendungen erscheinen müssen, ein solches äquivalentes Paar wurde leider nicht beobachtet.

35 Vgl. Eroms (2008:185-186).

36 Es erinnert teilweise an die Definitionsart ,genetisch-kausale Bestimmung“ bei Arntz/Picht/Schmitz (2014:64-65) bzw. „genetische oder operationale“ Definition bei Roelcke (2010:67). 
$\mathrm{Zu}$ den Gemeinsamkeiten gehört die Platzierung des Fachwortes im erklärenden Text. Das Fachwort kommt weder am Anfang noch am Ende der Textpassage, sondern jeweils in der Mitte der erklärenden Texte vor, sodass diese Fachwörter und deren Definitionen nicht so auffällig sind, als wenn sie sich einer der Definitionen nach Niederhauser (1999) hätten zuordnen lassen. Auf der anderen Seite kann sich der Rezipient dank des einleitenden Textes zuerst eine grobe Vorstellung machen und dann das Fachwort besser zu seinen schon existierenden Konzepten in seinem Gehirn einordnen. ${ }^{37}$

\section{Zusammenspiel des Verbalen und Bildlichen}

Beide Sendungen haben neben dem oben Genannten gemeinsam, dass sich die Pronomen das und toto/[das] nicht auf den vorangehenden Text beziehen, sondern es handelt sich um deiktische Ausdrücke, die den/die Rezipienten/Rezipientin auf das begleitende visuelle Material aufmerksam machen. ${ }^{38}$ In beiden Fällen werden Aufnahmen der besprochenen Tiere, d. h. authentische Videos aus der Natur, projiziert. ${ }^{39}$ Die allgemeine Problematik der Rollen in der Gruppe wird also in beiden Fällen an einem Beispiel des konkreten Affen-Männchens demonstriert.

\subsection{Typen der Definitionen im Vergleich}

Wie oben erwähnt wurde, lassen sich die Definitionen/Erklärungen nach verschiedenen Kriterien gliedern. Als grundsätzlich für die Gliederung der Unterkapitel wurde das Kriterium gewählt, ob auf das Fachwort oder auf die Definition explizit hingewiesen wird, oder nicht.

Es wurden zwei unterschiedliche Fälle beobachtet: entweder dienen einige Phrasen oder Verben zur Einleitung, oder fehlen diese, macht der Kommentator dagegen eine Pause in der Rede, was andeuten soll, dass der vorangehende oder nachkommende fachliche Ausdruck der entsprechenden Charakteristik gleichzusetzen ist. Man kann es als Ersatz des einleitenden Verbs ansehen und es kann also nicht mehr als implizit betrachtet werden. Auf der anderen Seite kann vorausgesetzt werden, dass dieser paraverbale explizite Hinweis andere Effekte bei Rezipienten/Rezipientinnen auslösen kann als ein verbaler Hinweis. ${ }^{40}$ Deswegen werden diese Beispiele in unterschiedlichen Unterkapiteln vorgelegt.

Zusätzlich wurden die Definitionen/Erklärungen nach weiteren Kriterien analysiert, die in Kapitel 3 genannt wurden (vor allem Typen der Definition nach der Reihenfolge der Erklärung und der definierten Fachwörter). In den letzten zwei Unterkapiteln werden darüber hinaus zwei Spezifika behandelt: Definitionen/Erklärungen, die auf die Ursache der Benennung hinweisen, und Passagen, in denen mehrere Typen der Definitionen kombiniert auftauchen, indem mehrere Fachwörter vorgestellt werden. Gemeinsame und unterschiedliche Merkmale werden wieder in einzelnen Tabellen festgehalten.

\subsubsection{Definitionen ohne explizite Einleitung}

Sowohl kataphorische als auch anaphorische Definitionen können in den Text sehr unauffällig eingeschaltet werden (siehe Tabelle 3).

\begin{tabular}{|l|l|}
\hline $\begin{array}{l}\text { K / D } \\
\text {,Lexikon` }\end{array}$ & $\begin{array}{l}\text { Beispiel } 5 \\
{[\ldots] \text { Mit der Zeit sammelten sich immer mehr Pflanzenreste am Grund an. Die Seen wurden }} \\
\text { flacher und flacher und die Pflanzen vom Ufer konnten sich nun weiter ausbreiten. Die Seen } \\
\text { verlandeten. }[\ldots] \\
\text { URL } 27\end{array}$ \\
\hline
\end{tabular}

\footnotetext{
37 Vgl. Göpferich (2001: 130-132).

38 Komplementäre Beziehung des Textes und des visuellen Begleitmaterials (vgl. Stöckl 2004: 250).

39 D. h. Typ des Bildes: Abbild (vgl. Weidenmann 1994: 12-25), Ikone (vgl. Burger/Luginbühl 2014: 413-415).

40 Es muss bedacht werden, dass die Rezipienten/Rezipientinnen die Texte (bis auf Ausnahmen) nicht sehen, sondern nur hören.
} 


\begin{tabular}{|c|c|}
\hline $\begin{array}{l}\text { E / D } \\
\text {,Wilde Welt }\end{array}$ & $\begin{array}{l}\text { Beispiel } 6 \\
{[\ldots] \text { Ein virtuoser Flieger ist der Papageitaucher nicht. }[\ldots] \text { Er ist ein Taucher. Mit einem }} \\
\text { Atemzug kommt er siebzig Meter weit. Wie Pinguine nutzt er seine Flügel zum Schwimmen. } \\
{[\ldots]} \\
\text { URL } 13\end{array}$ \\
\hline $\begin{array}{l}\mathrm{K} / \mathrm{T} \\
\text {,Ptačí } \\
\text { rodinky“ }\end{array}$ & $\begin{array}{l}\text { Beispiel } 7 \\
\text { [...] U špačků vládne rovnoprávnost. Stejně jako se špačči rodiče spravedlivě střídali při } \\
\text { sezeni na vajičkách, střídaji se i při krmeni svých zlatícek. [...] } \\
\text { [Bei Staren regiert die Gleichberechtigung. Gleich wie sich die Star-Eltern gerecht beim } \\
\text { Brüten abgewechselt haben, wechseln sie sich auch bei der Fütterung ihrer Goldkinder ab.] } \\
\text { URL } 6 \text { (Serienteil: Špaččí rodinka/[Kleine Star-Familie]) }\end{array}$ \\
\hline $\begin{array}{l}\text { E / T } \\
\text {,Primáti‘ }\end{array}$ & $\begin{array}{l}\text { Beispiel } 8 \\
\text { [...] Pro tyto mladé orangutany je stěžejní, aby si dokázali informace zapamatovat a později } \\
\text { byli schopni si je vybavit. Mají epizodickou pamět' stejně jako lidé. Propojuje se jim, co, } \\
\text { kdy a kde se odehrálo. Inteligence je založena na vzpominkách, které tvoři takový mozkový } \\
\text { archiv. [...] } \\
\text { [Für diese jungen Orang-Utans ist essenziell, dass sie sich die Informationen merken können } \\
\text { und fähig sind, diese später abzurufen. Sie haben ein episodisches Gedächtnis genauso wie } \\
\text { die Menschen. Sie verbinden, was, wann und wo sich etwas ereignet hat. Die Intelligenz } \\
\text { beruht auf Erinnerungen, die ein solches Gehirnarchiv bilden.] } \\
\text { URL19 }\end{array}$ \\
\hline
\end{tabular}

Tab. 3: Definitionen ohne explizite Einleitung

Anmerkung: $\mathrm{K}=$ Fernsehsendung für Kinder, $\mathrm{E}=$ Fernsehsendung für Erwachsene, $\mathrm{D}=$ deutsche Fernsehsendung, $\mathrm{T}=$ tschechische Fernsehsendung

Wie die Beispiele belegen, können die Kommentare mit dem implizit eingeführten Fachwort auf verschiedene Art und Weise gestaltet werden.

Das Beispiel 5 stellt eine implizite kataphorische Definition/Erklärung des Verbs verlanden bzw. eine Begriffskondensation ${ }^{41}$ als Mittel der Kohäsion dar. ${ }^{42}$ Die sukzessive Entwicklung und eine gewisse Intensivierung wird mithilfe der Wiederholung des Worts flacher skizziert. ${ }^{43}$ Zum Einprägen bei den Rezipienten/Rezipientinnen können auch gleiche Anfänge der Sätze (die Seen) beitragen (Anapher). ${ }^{44}$

Die Beispiele 6 und 7 folgen einem ähnlichen Prinzip, obwohl ersteres aus der Fernsehsendung für Kinder und letzteres für Erwachsene stammen. Die Fachwörter werden durch eine anaphorische Definition/Erklärung näher gebracht, wobei man in der Fernsehsendung für Erwachsene (Beispiel 6) auf die Angabe der konkreten Fähigkeiten des Vogels und den Vergleich mit dem wohl bekannteren Vogel (Pinguin) setzt. Beim Beispiel 7 wird im weiteren Satz nach der Angabe des Fachwortes rovnoprávnost/[Gleichberechtigung] ausgeführt, was damit konkret gemeint wird (Topik durch Paraphrase ${ }^{45}$ bzw. Exemplifizierung). Es wird vorausgesetzt, dass sich die Kinder des Zusammenhangs mithilfe der semantisch verbundenen Wörter rovnoprávnost/[Gleichberechtigung] - spravedlivěl [gerecht]) bewusst werden. ${ }^{46}$

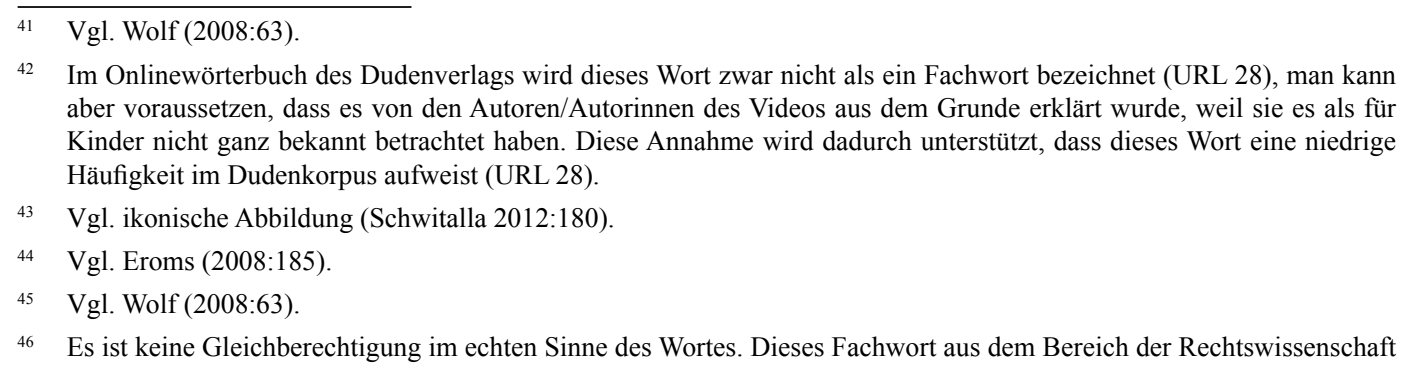


Des Weiteren ist beim Beispiel 8 aus der Fernsehsendung für Erwachsene interessant, dass es sich hier um eine Erklärung bzw. Definition einer terminologischen Wortverbindung handelt, von der ein Teil kataphorisch (pamét'/[Gedächtnis]) und ein Teil anaphorisch (epizodická/[episodisch]) definiert wird. Es kann vorausgesetzt werden, dass eine solche Textgestaltung einen größeren kognitiven Aufwand erfordert, sodass die Verwendung in einer Sendung für Erwachsene entsprechend ist. In dem weiteren erklärenden Satz kann die Metapher mozkový archiv/[Gehirnarchiv] die Situation besser veranschaulichen bzw. den Text interessanter machen. ${ }^{47}$

\section{Zusammenspiel des Verbalen und Bildlichen}

Die Relevanz des bildlichen Materials ist unterschiedlich, je nach dem, wie sehr sich es an dem Verständnis beteiligt oder beteiligen kann. Von Belang ist es bei den Textpassagen 5 und 6, weil das Verständnis der Informationen ohne Bild zwar nicht unmöglich wäre, jedoch verzerrt sein könnte. Die Animation im Beispiel 5 zeigt den ganzen Prozess der Verlandung in einzelnen Schritten und das Video im Beispiel 6 garantiert, dass sich die Rezipienten/Rezipientinnen die Bewegungen des Vogels nicht falsch vorstellen. ${ }^{48}$

Bei den Beispielpassagen 7 und 8 können Beziehungen des Kommentars zum visuellen Begleitmaterial (reale Videos aus der Natur mit den besprochenen Tieren) als redundant bezeichnet werden. Einerseits kann angenommen werden, dass die Kinder schon einige Erfahrungen oder eventuell Erlebnisse im Zusammenhang mit der Fütterung der Vögel haben, andererseits zeigt das Video bei diesem Satz nicht direkt die Fütterung, sondern nur zwei Stare, die Futter suchen und tragen. Noch weniger wichtig ist das Video beim Kommentar 8, das nur eine illustrative Funktion ausübt, indem Orang-Utans in der Natur und die sie beobachtenden Wissenschaftlerinnen gezeigt werden. Insgesamt spielt das Video aber bei dieser ganzen Passage zum Thema „Orang-Utans“ aus der jeweiligen Fernsehsendung eine sehr wichtige Rolle, weil vor diesem als Zusammenfassung geltenden Kommentar konkrete Tätigkeiten und Fähigkeiten von Orang-Utans demonstriert und kommentiert wurden, die von ihrer hohen Intelligenz zeugen, was für das gute Verständnis der Rezipienten/Rezipientinnen also unentbehrlich ist.

\subsubsection{Definitionen mit einer paraverbalen Einleitung}

Die paraverbalen Mittel - vor allem Pausen ${ }^{49}$ in der Rede - können Fachwörter oder Definitionen/ Erklärungen in den analysierten Fernsehsendungen sowohl für deutsche und tschechische Kinder als auch für deutsche und tschechische Erwachsene signalisieren (siehe Tabelle 4). ${ }^{50}$

\begin{tabular}{|l|l|}
\hline $\begin{array}{l}\text { K / D } \\
\text { Tierbabys`‘ }\end{array}$ & $\begin{array}{l}\text { Beispiel } 9 \\
{[\ldots] \text { Sie sind von Kopf bis zu den Füßen über und über mit einem weichen, braunen, }} \\
\text { fluffigen Flaum bedeckt - den Daunen. Das ist vielleicht nicht sehr schick, aber dafür sehr, } \\
\text { sehr warm. }[\ldots] \\
\text { URL } 22\end{array}$
\end{tabular}

wird in diesem Zusammenhang vielmehr metaphorisch bzw. zur Annäherung und zum Vergleich mit der Menschenwelt verwendet.

47 Diese Definition erinnert an die Definitionsart „Funktionsangabe“ bei Arntz/Picht/Schmitz (2014:64-65).

48 Abbilder als Typen der Bilder; komplementäre Beziehung zwischen dem Kommentar und dem bildlichen Material.

49 Vgl. syntaktische Fragmentierung in mündlichen Texten bei Schwitalla (2012:110-117).

50 Dieses nicht ausgesprochene „Gleichheitszeichen“ würde im geschriebenen Text einem Gedankenstrich (bzw. einem Doppelpunkt) entsprechen. Da es sich um eine Transkription der mündlichen Kommentare handelt, kann die Typografie in diesen auf verschiedene Art und Weise interpretierbaren Fällen nicht mit Sicherheit eingesetzt werden. Die Autorin hat sich für Gedankenstriche entschieden. 


\begin{tabular}{|c|c|}
\hline $\begin{array}{l}\text { E / D } \\
\text {,Wilde Welt }\end{array}$ & $\begin{array}{l}\text { Beispiel } 10 \\
{[\ldots] \text { Die Lachswanderung in die großen Flüsse beginnt erst ab August. Diese vegetarische }} \\
\text { Kost dient der Überbrückung. Segge - ein Sauergrasgewächs. Relativ kalorienarm, dafür } \\
\text { aber reich an wertvollen Mineralien. [...] } \\
\text { URL } 15\end{array}$ \\
\hline $\begin{array}{l}\mathrm{K} / \mathrm{T} \\
\text {,Kvítí }\end{array}$ & $\begin{array}{l}\text { Beispiel } 11 \\
\text { [...] U země zelené listy, rozprostřené dokola, jako plesová sukýnka. Vyrůstají z jednoho } \\
\text { mista - trsu. [...] } \\
\text { [Am Boden grüne Blätter, rundherum ausgebreitet, wie ein Ballröckchen. Sie wachsen an } \\
\text { einem Platz - Büschel.] } \\
\text { URL } 5 \text { (Serienteil: ,Sedmikráska'/[Gänseblümchen]) }\end{array}$ \\
\hline $\begin{array}{l}\mathrm{E} / \mathrm{T} \\
\text {,Primáti“ }\end{array}$ & $\begin{array}{l}\text { Beispiel } 12 \\
{[\ldots] \text { Genetické předpoklady - vyvinutý mozek, oči vpředu a chápavá ruka -jsou tedy }} \\
\text { klićem k úspěchu celého ŕádu primátù. [...] } \\
\text { [Genetische Voraussetzungen - ein entwickeltes Gehirn, Augen vorne und eine Greifhand - } \\
\text { sind also Schlüssel zum Erfolg der gesamten Ordnung der Primaten.] } \\
\text { URL } 17\end{array}$ \\
\hline
\end{tabular}

Tab. 4: Definitionen mit einer paraverbalen Einleitung

Anmerkung: $\mathrm{K}=$ Fernsehsendung für Kinder, $\mathrm{E}=$ Fernsehsendung für Erwachsene, $\mathrm{D}$ = deutsche Fernsehsendung, $\mathrm{T}=$ tschechische Fernsehsendung

Eine solche Gestaltung von Definitionen macht den Text dynamischer und das Fachwort wird durch diese akustische Abgrenzung vom restlichen Text hervorgehoben.

Bei dem Beispiel 9 wird die Dynamik und Intensität des Ausdrucks noch durch die asyndetisch verbundenen (auch umgangssprachlichen) Attribute (weichen, braunen, fluffigen) erhöht. ${ }^{51}$ Im weiteren Kommentar werden auch Wiederholungen zur Intensivierung genutzt (sehr, sehr). ${ }^{52}$

Bei dem Beispiel 10 handelt es sich um eine Folge von zwei Fachwörtern, wobei das erste Fachwort durch ein anderes, allgemeineres Fachwort mit einer durchschaubaren lexikalischen Motivation nach der Pause erklärt bzw. umschrieben wird.$^{53}$ Erst danach wird die Charakteristik dieser Pflanze durch die nahrhaften Angaben aus der Perspektive der fressenden Tiere weiter ausgeführt.

Das Prinzip der Textgestaltung im Beispiel 11 aus der tschechischen Fernsehsendung für Kinder ähnelt dem Prinzip im Beispiel 9 für deutsche Kinder, zusätzlich wird hier eine Stilfigur des Vergleichs (jako plesová sukýnka/[wie ein Ballröckchen]) ${ }^{54}$ verwendet, um die Attraktivität des Textes für Kinder zu steigern. Der erste Satz, der sich an der Charakteristik beteiligt, ist elliptisch konzipiert, ${ }^{55}$ sodass die Beschreibung nicht weitschweifig wirkt. ${ }^{56}$

Einen speziellen Status nimmt die Definition im Beispiel 12 aus der tschechischen Fernsehsendung für Erwachsene ein. Es handelt sich um eine Zusammenfassung von davor einzeln beschriebenen Spezifika der Primaten, die in die terminologische Wortverbindung genetische Voraussetzungen eingeschlossen werden. Um ein besseres Merken zu sichern, werden diese einzelnen Merkmale

51 Diese Definition erinnert an die Definitionsart „Angaben zur materiellen Beschaffenheit“ bei Arntz/Picht/Schmitz (2014:64-65). Es handelt sich um eine kataphorische Definition.

52 Vgl. ikonische Abbildung (Schwitalla 2012:180). Vgl. das Beispiel 5 im Unterkapitel 5.2.1.

53 Es handelt sich um eine anaphorische Definition. Die Segge gehört zur Familie der Sauergrasgewächse (Cyperaceae) (vgl. Haller/Probst 1989:141).

54 Vgl. Eroms (2008:182).

55 Eroms (2008:190) zählt Ellipsen zu Stilfiguren, Malá (2009:52-54) zu abweichenden Satzkonstruktionen.

56 Vgl. Problematik der Textverständlichkeit - Prägnanz (Göpferich 2001:126-128). Es handelt sich um eine kataphorische Definition. 
durch Aufzählung ${ }^{57}$ wiederholt (worauf das Konjunktionaladverb tedy/[also] hindeutet) und akustisch vom Satz als eine elliptische Parenthese abgegrenzt. ${ }^{58}$ Die erste Pause könnte durch einen verbalen Ausdruck ersetzt oder ergänzt werden (z. B. „das heißt“). Man kann hier also eine Kombination der kataphorischen (einführenden und ausführlichen) Definition ${ }^{59}$ und der anaphorischen (kurz gefassten, wiederholenden) Definition beobachten.

\section{Zusammenspiel des Verbalen und Bildlichen}

Die analysierten Textpassagen 9, 10, 12 werden mit einem realen Video aus der Natur mit den beschriebenen Tieren und Pflanzen begleitet, die Textpassage 11 ist mit einer Animation der Blätter verbunden. Die Kommentare wären auch ohne das visuelle Begleitmaterial verständlich (redundante Beziehung), diese Bezeichnung ,redundant“ im eigentlichen Sinne des Wortes wäre jedoch bei beschriebenen Fällen irreführend, denn die begleitenden Videos und Animation sind nicht ganz redundant bzw. irrelevant und dienen einer besseren Vorstellung und damit einem besseren Merken sowie der Motivation. ${ }^{60}$

\subsubsection{Definitionen mit einer verbalen Einleitung}

Fachwörter, die selbst oder deren Definitionen explizit eingeleitet werden, sodass die Aufmerksamkeit der Rezipienten/Rezipientinnen darauf intensiver gelenkt werden kann, kann man sowohl in den analysierten Fernsehsendungen für Kinder als auch für Erwachsene finden. Entweder handelt es sich um ganze einleitende Sätze, oder nur um Verben, die Bestandteil der Definition bilden und die Inhalte wie Nennen, Bedeuten oder Erkennen usw. ausdrücken. ${ }^{61}$ Diese Hinweise sind entweder „unpersönlich “62 formuliert, oder man spricht dadurch den Rezipienten direkt an. Da hier viele Möglichkeiten der Ausdrücke bestehen, werden in diesem Unterkapitel verschiedene Typen der Ausdrücke exemplarisch vorgestellt (siehe Tabelle 5).

\begin{tabular}{|l|l|}
\hline $\begin{array}{l}\text { K / D } \\
\text { Tierbabys }\end{array}$ & Siehe Beispiel 3 \\
\hline $\begin{array}{l}\text { E / D } \\
\text {,Wilde Welt }\end{array}$ & $\begin{array}{l}\text { Beispiel 13 } \\
{[\ldots] \text { Sie sind effiziente Jäger und wie viele ihrer Artgenossen sehr neugierig. Kleine }} \\
\text { Schwertwale. Auch falsche Killerwale genannt. }[\ldots] \\
\text { URL } 14\end{array}$ \\
\hline
\end{tabular}

57 Vgl. Eroms (2008:185).

58 Es erinnert an die ,exemplarische“ Definition bei Roelcke (2010:65).

59 Vgl. Begriffskondensation bei Wolf (2008:63). Vgl. das Beispiel 8 im Unterkapitel 5.2.1.

60 Unter klassischen Umständen wäre das Video von der Pflanze „Segge“ relevant, damit der Rezipient sieht, welchen Aufbau die Pflanze hat, und diese dann in der Natur besser identifizieren kann. In der analysierten Sendung geht es jedoch primär nicht um den Aufbau der erwähnten Pflanze, sondern darum, dass es eine Nahrung für Bären ist, die das Hauptthema der Sendung darstellen. Deswegen spielt das genaue Bild der Pflanze eine nicht so große Rolle (dem entspricht keine detaillierte Aufnahme der Pflanze), für eine volle Skizzierung des Lebensraumes und der Lebensweise von Bären ist es in gröberen Zügen vonnöten.

Bei dem Beispiel 12 sind die Videoaufnahmen der Affen vor dem zusammenfassenden Kommentar relevant, weil dadurch die einzelnen Merkmale und Fähigkeiten der Primaten demonstriert werden. Bei der Zusammenfassung ist also das bildliche Material nicht mehr unbedingt notwendig und auch nicht mehr so genau auf die einzelnen Merkmale konzentriert wie bei der vorangehenden Beschreibung.

${ }^{61}$ Vgl. Definitor bei der klassischen, sog. aristotelischen Definition (Roelcke 2010:62).

62 Diese Unterscheidung wird aus der Perspektive der Rezipienten/Rezipientinnen durchgeführt. Mit dieser Bezeichnung „unpersönlich“ wird hier die Ausdrucksweise verstanden, durch die die Tatsachen allgemein beschrieben und die Rezipienten/Rezipientinnen dabei nicht in Betracht gezogen und/oder angesprochen werden (die verschiedenen Typen des Passivs usw. werden nicht berücksichtigt). 


\begin{tabular}{|c|c|}
\hline & $\begin{array}{l}\text { Beispiel } 14 \\
\text { [...] Und hier ist er zu Hause, der Waldmensch, wie ihn die Indonesier nennen. [...] } \\
\text { URL } 13\end{array}$ \\
\hline & $\begin{array}{l}\text { Beispiel } 15 \\
\text { [...] Orang-Utans leben solitär. Das heißt, es gibt keine großen Gruppen, sondern immer } \\
\text { nur kleine einzelne Tiere. [...] } \\
\text { URL } 13\end{array}$ \\
\hline $\begin{array}{l}\text { K / T } \\
\text {,Kvítí }\end{array}$ & $\begin{array}{l}\text { Beispiel } 16 \\
\text { [...] Sedmikráska má moc ráda slunce, takže za ním obrací hlavičku. Ǩíká se tomu, že je } \\
\text { heliotropní, stejně jako napřiklad slunečnice. [...] } \\
\text { [Das Gänseblümchen mag die Sonne sehr, sodass es zu ihr sein Köpfchen dreht. Es heißt, } \\
\text { dass sie „heliotrop“ ist, genauso wie zum Beispiel die Sonnenblume.] } \\
\text { URL } 5 \text { (Serienteil: ,Sedmikráska‘/[Gänseblümchen]) } \\
\text { Beispiel } 17 \\
\text { [...] Poznávací znamení heřmánku pravého jsou: specifická vioně,, nahořklá chut’ a pře- } \\
\text { devším duté lưžko květu. Poznáme to tak, že utrhneme jeden květ a nehtem ho rozdělime. } \\
\text { Když je dutý, je to heřmánek. [...] } \\
\text { [Die Erkennungszeichen der Echten Kamille sind: spezifischer Duft, bitterlicher Ge- } \\
\text { schmack und vor allem ein hohles Bett der Blüte. Wir erkennen es so, dass wir eine Blüte } \\
\text { abreißen und mit dem Nagel teilen. Wenn sie hohl ist, ist es Kamille.] } \\
\text { URL } 5 \text { (Serienteil: „Heřmánek‘/[Kamille]) }\end{array}$ \\
\hline $\begin{array}{l}\mathrm{K} / \mathrm{T} \\
\text {,Ptačí rodinky“ }\end{array}$ & $\begin{array}{l}\text { Beispiel } 18 \\
\text { [...] Poštolka obecná je dravec přibuzný sokolům. [...] } \\
\text { [Der Turmfalke ist ein Raubvogel, verwandt mit Falken.] } \\
\text { URL } 6 \text { (Serienteil: ,Poštolčí zásnuby‘/[Verlobung der Turmfalken]) }\end{array}$ \\
\hline $\begin{array}{l}\text { E / T } \\
\text {,Primáti‘ }\end{array}$ & Siehe Beispiel 4 \\
\hline
\end{tabular}

Tab. 5: Definitionen mit einer verbalen Einleitung

Anmerkung: $\mathrm{K}=$ Fernsehsendung für Kinder, $\mathrm{E}=$ Fernsehsendung für Erwachsene, $\mathrm{D}=$ deutsche Fernsehsendung, $\mathrm{T}=$ tschechische Fernsehsendung

\section{Unpersönliche Formulierungen}

Die unpersönlichen Formulierungen tauchen sowohl in Fernsehsendungen für Erwachsene als auch für Kinder auf, wie den Beispielen 13 (durch Passiv, siehe auch das Beispiel 3 im Unterkapitel 5.1.2. und Beispiel 19 im Unterkapitel 5.2.4.), 15 (durch die allgemeine Phrase das heißt), 16 (die reflexive Form des Verbs) ${ }^{63}$ und 18 (nur das bloße Verb sein) ${ }^{64} \mathrm{zu}$ entnehmen ist. Der einzige Unterschied (neben dem Unterschied in der Reihenfolge der Angaben), der beim Vergleich gerade dieser Beispiele auffällt (was jedoch nicht verallgemeinernd sein kann), ist, dass die Beschreibung/Erklärung für Kinder in gewissem Maße metaphorisch ist, sei es der Ausdruck, dass das Gänseblümchen etwas mag, oder der Ausdruck, dass sie zur Sonne sein Köpfchen dreht. Diese Metapher könnte Vorschulkinder ein bisschen irreführen, aber durch die den Kommentar begleitende Animation, in der die Bewegungen der Blumen angedeutet werden, wird klar, dass kein echter Kopf, sondern die Blüte der Blume gemeint ist (zum visuellen Begleitmaterial siehe unten). ${ }^{65}$

63 Im Unterschied zum Deutschen ist der angegebene Ausdruck für die Benennung (Říká se tomu) im Tschechischen reflexiv.

64 Es erinnert an die Definitionsart „taxonomisch-nominale Begriffserklärung“ bei Arntz/Picht/Schmitz (2014:64-65) bzw. klassische, sog. aristotelische Definition (Roelcke 2010:62).

65 Komplementäre Beziehung des Textes und des Bildes (vgl. Stöck1 2004:250). 
An dem Beispiel 13 aus der Sendung für Erwachsene ist noch spezifisch, dass die Information über die Benennung als ein Nachtrag angegeben ist (kataphorische Definition). Zuerst wird das beschriebene Objekt mit einem Pronomen bezeichnet (sie), erst danach erscheinen die Namen der Tiere am Ende der Textpassage, wodurch eine gewisse Spannung hervorgerufen wird. Der Ausdruck der Benennung ist darüber hinaus elliptisch verfasst (die erste Benennung der Tiere wird durch kein Verb eingeleitet, im zweiten Satz mit der alternativen Benennung fehlt ein Hilfsverb bei der Passivform). Dadurch kann der Text dynamischer wirken. Diese Gestaltung des Textes erfordert für das volle Verständnis eine unbedingte Begleitung des visuellen Materials (in diesem Falle ist das ein Video mit Schwertwalen im Meer), denn auf das Tier wird deiktisch hingewiesen (siehe unten). ${ }^{66}$

\section{Persönliche Formulierungen}

Die persönlichen Formulierungen von einleitenden Ausdrücken befinden sich genauso in Fernsehsendungen für beide analysierten Altersgruppen. Das Beispiel 14 aus der deutschen Fernsehsendung für Erwachsene basiert darauf, dass man konkrete Personen erwähnt, die etwas irgendwie benennen (siehe auch Beispiel 22 im Unterkapitel 5.2.4.). Darüber hinaus wird hier ein ähnliches Prinzip wie im Beispiel 13 befolgt, d. h., zuerst ein deiktischer Hinweis auf das Tier im Video, erst danach die Benennung des Tieres, in diesem Falle in der Apposition. ${ }^{67}$ In der analysierten tschechischen Fernsehsendung für Erwachsene (siehe Beispiel 4 im Unterkapitel 5.1.2.) wurde eine Form des Verbs beobachtet, durch die sich der Kommentator an die Zuschauer/Zuschauerinnen wendete (Podle ní poznáte/[Danach erkennen Sie]).

\section{Tschechische Formulierungen mit einem allgemeinen Subjekt my/[wir]}

Einen speziellen Status und einen gewissen Zwischenstand stellen Bezeichnungen im Tschechischen dar, die eine universelle Bezeichnung wir verwenden (siehe Beispiel 17 aus der Fernsehsendung für Kinder, anaphorische Definition). Es handelt sich um ein allgemeines Subjekt zur Formulierung der allgemeinen Tatsachen. Ein Äquivalent im Deutschen ist die Formulierung mit „man“. Weil dazu aber kein spezielles unpersönliches Pronomen verwendet wird wie im Deutschen, sondern ein Personalpronomen, das normalerweise auf eine persönliche Formulierung hindeutet, können solche Formulierung persönlicher wirken und der/die Zuschauer/Zuschauerin kann sich mehr angesprochen und in die Forschung miteingeschlossen fühlen als z. B. bei der Formulierung mit einer Passivform. Dank der Animation, die ganz genau nahelegt, welcher Teil der Blüte geteilt werden soll, muss nicht näher sprachlich erklärt werden, was mit dem Bett der Blüte gemeint ist.

\section{Zusammenspiel des Verbalen und Bildlichen}

Wie aus den oben angegebenen Analysen folgt, ist das visuelle Begleitmaterial bei den meisten Beispielen unentbehrlich, weil die Charakteristik unbedingt auf das Video oder Amination beruht und deswegen auch kürzer und bündiger sprachlich konzipiert werden kann als ohne Bildbegleitung. Umgekehrt wäre das visuelle Begleitmaterial selbst ohne Kommentar schwierig verständlich, denn man wüsste nicht, worauf man sich konzentrieren soll und was die Bilder mitteilen wollen. ${ }^{68}$ Lediglich bei der Beispielpassage 15 könnte man eventuell auf das begleitende visuelle Material verzichten, ohne dass der Text unklar wäre, weil die Beschreibung eindeutig ist und diese Problematik kein großes Vorstellungsvermögen erfordert. ${ }^{69}$

\footnotetext{
66 Diese Definition erinnert an die Definitionsart „Angabe allgemeiner Merkmale“ bei Arntz/Picht/Schmitz (2014:64-65) und Synonymendefinition nach Roelcke (2010:68).

67 Vgl. Malá (2009:52-54), die Appositionen zu abweichenden Satzkonstruktionen zählt.

68 Komplementäre Beziehung (vgl. Stöckl 2004:250).

69 Redundante Beziehung (vgl. Stöckl 2004:250).
} 


\subsubsection{Definitionen mit Ursache der Benennung (Motivation)}

Definitionen und Erklärungen, die die Ursache der Benennung (Motivation) explizit machen und so zum Verständnis beitragen können, können sowohl in den Fernsehsendungen für Kinder als auch für Erwachsene beobachtet werden. Bei der tschechischen Sendung für Kinder wurde eine solche Definition schon in dem Beispiel 2 (Unterkapitel 5.1.1.) beschrieben (nektarodárný/[Nektar gebend]). Weitere Beispiele werden in der Tabelle 6 geboten.

\begin{tabular}{|c|c|}
\hline $\begin{array}{l}\text { K / D } \\
\text {,Tierbabys }\end{array}$ & $\begin{array}{l}\text { Beispiel } 19 \\
{[\ldots] \text { Ihre Heimat-Tibet-wird auch das Dach der Welt genannt, wegen der hohen Berge }} \\
\text { dort. Mutter und Vater sehen gleich aus, sie haben schwarze Streifen auf dem Kopf. Deshalb } \\
\text { heißen sie auch Streifengänse. [...] } \\
\text { URL } 23\end{array}$ \\
\hline $\begin{array}{l}\text { E / D } \\
\text {,Wilde Welt }\end{array}$ & $\begin{array}{l}\text { Beispiel } 20 \\
{[\ldots] \text { Eineinhalb Kilogramm Fisch täglich verspeist ein erwachsener Grauskopfpelikan. Ihr }} \\
\text { strobliger Schopf-der Namensgeber. [...] } \\
\text { URL } 14\end{array}$ \\
\hline $\begin{array}{l}\mathrm{K} / \mathrm{T} \\
\text {,Kvítí‘ }\end{array}$ & $\begin{array}{l}\text { Beispiel } 21 \\
\text { [...] Šedozelené listy jsou střidavé, úzce kopinaté. To znamená, že jsou úzké a dlouhé, } \\
\text { trochu pripominaji kopí, proto kopinaté. [...] } \\
\text { [Graugrüne Blätter sind abwechselnd angeordnet, schmal lanzettförmig. Das bedeutet, dass } \\
\text { sie schmal und lang sind, ein bisschen erinnern sie an eine Lanze, deswegen lanzenförmig.] } \\
\text { URL } 5 \text { (Serienteil: ,Chrpa‘/[Kornblume]) }\end{array}$ \\
\hline $\begin{array}{l}\mathrm{E} / \mathrm{T} \\
\text {,Primáti“ }\end{array}$ & $\begin{array}{l}\text { Beispiel } 22 \\
\text { Toto je samice tamarina vousatého. Vědci ji pojmenovali ,RC“, což je v angličtině zkratka } \\
\text { pro obojek s vysílačkou. } \\
\text { [Das ist ein Weibchen des Kaiserschnurrbarttamarins. Die Wissenschaftler/Wissenschaft- } \\
\text { lerinnen haben es „RC“ benannt, was im Englischen eine Abkürzung für ein Halsband mit } \\
\text { dem Sender ist.] } \\
\text { URL } 18\end{array}$ \\
\hline
\end{tabular}

Tab. 6: Definitionen mit Ursache der Benennung (Motivation)

Anmerkung: $\mathrm{K}=$ Fernsehsendung für Kinder, $\mathrm{E}=$ Fernsehsendung für Erwachsene, $\mathrm{D}$ = deutsche Fernsehsendung, $\mathrm{T}=$ tschechische Fernsehsendung

Im Beispiel 19 aus der deutschen Sendung für Kinder ,Tierbabys“ wird zuerst die Motivation der Benennung der Vögel angegeben (schwarze Streifen), erst danach folgt die Bezeichnung selbst (kataphorische Definition). Der Grund für diese Bezeichnung wird noch mithilfe des Konjunktionaladverbs deshalb explizit gemacht. Davor kann man noch eine Charakteristik eines von diesen Gänsen bewohnten Landes beobachten, wobei die Bezeichnung des Landes vom Rest des Textes durch Pausen abgegrenzt und dadurch hervorgehoben ist (vgl. Unterkapitel 5.2.2.). ${ }^{70}$ Dann wird die andere, metaphorische Bezeichnung des Landes mithilfe der expliziten Information über die Bezeichnung (der Satz mit dem Prädikat nennen im Passiv) beigefügt. Auf die lexikalische Motivation der Bezeichnung wird in der Ausklammerung explizit hingewiesen, diesmal mithilfe der kausalen Präposition wegen.

Das gleiche Prinzip, dass die Bezeichnung des Tieres durch den Hinweis auf dessen Aussehen erklärt wird, wird im Beispiel 20 aus der deutschen Fernsehsendung für Erwachsene genutzt. Hier

$70 \quad$ In der Transkription kann es durch Gedankenstriche oder Kommas (als Apposition) angedeutet werden. Weil es sich um eine konkrete Ergänzung zur allgemeinen Bezeichnung ihre Heimat handelt, die auch mithilfe eines Prädikats eingeleitet werden könnte (,das ist Tibet“), wurden Gedankenstriche zur Andeutung der elliptischen Parenthese ausgewählt. 
begrenzt sich der Kommentar auf einen elliptischen Satz, in dem das Kopula-Verb mithilfe einer Pause ersetzt wird (vgl. Unterkapitel 5.2.2.). Zugleich wird das in Frage kommende einleitende Verb „nennen“ oder „bezeichnen als“ bzw. „den Namen geben“ durch einen substantivierten Ausdruck ersetzt (der Namensgeber), sodass der ganze Satz kurz und bündig wird. Im Vergleich zum Beispiel 19 sind die Informationen umgekehrt strukturiert als bei Streifengänsen und gleich wie bei Tibet, d. h., zuerst wird die Bezeichnung angegeben, dann die Motivation der Bezeichnung selbst.

Eine „kombinierte“ Strategie (anaphorisch-kataphorische Definition) kann man bei dem Beispiel 21 aus der tschechischen Fernsehsendung für Kinder beobachten. Zuerst wird das Fachwort genannt, danach folgt die Erklärung der Motivation des Fachwortes (siehe To znamená/[Das bedeutet]) und dann wird das Fachwort in einem elliptischen Satz wiederholt, wobei der Grund der Bezeichnung noch durch das Konjunktionaladverb proto/[deshalb] explizit angedeutet wird. Der Aufbau der Passage erinnert an Alltagskommunikation, d. h. eine scheinbar nicht völlig durchdachte Beschreibung mit Redundanzen, was wahrscheinlich den Zweck hat, dass der begleitende Kommentar nicht zu ,schulmeisterlich“" wirken soll.

Von den oben angegebenen Beispielen unterscheidet sich das Beispiel 22. Der Kommentar gibt keine Information zum Grund für eine offizielle Benennung eines Tieres wieder, sondern es wird erklärt, aus welchem Grund die Wissenschaftler/Wissenschaftlerinnen das Weibchen des Kaiserschnurrbarttamarins einen inoffiziellen Namen gegeben haben (es kann also als ein gewisser Jargon bezeichnet werden).71 Diese Information ist für die Zuschauer/Zuschauerinnen eigentlich nicht von Belang, aber sie können eine persönlichere Beziehung zum Tier entwickeln, indem nicht mehr der offizielle biologische Name bei der Beschreibung wiederholt wird, sondern der Eigenname des Tieres.

\section{Zusammenspiel des Verbalen und Bildlichen}

Weil in den meisten genannten Fernsehsendungen das äußere Aussehen der Tiere oder Pflanzen erklärt werden, ist das visuelle Begleitmaterial von hoher Relevanz (Beispiele 19-21).$^{72}$ Am relevantesten kann die Aufnahme der Vögel im Beispiel 20 betrachtet werden, weil man mit der kurz verfassten Beschreibung ,strobliger Schopf“ verschiedene Vorstellungen assoziieren kann. Im Beispiel 19 ist die Beschreibung länger, trotzdem ist das begleitende Video aus der Umgebung der Berge mit der Aufnahme der Vögel wichtig, denn die Streifen könnten von den Kindern sonst auf unterschiedliche Art und Weise vorgestellt werden. Im Beispiel 21 wird der Benennungsgrund mit der Skizze (Animation) angedeutet - dabei wird während des Kommentars schrittweise das schmale Blatt gezeichnet. Eine Lanze selbst wird jedoch dabei zum Vergleich nicht gezeichnet. Wohl vermuten die Autoren/Autorinnen, dass sich die Kinder diese Waffe vorstellen können. Lediglich beim Beispiel 22 braucht man für die konkrete Information kein Bild, weil es sich um eine zusätzliche Information handelt. ${ }^{73}$

\subsubsection{Kombinierte Definitionen, Gliederung des Textes}

Die Definitionen und Erklärungen von Fachwörtern sind oft nicht isoliert, sondern meistens werden mehrere Fachwörter in einer Textpassage im Kontrast erklärt. Das ist mit dem Bedürfnis verbunden, den ganzen Text systematisch zu gliedern. Dagegen mussten die Autoren/Autorinnen in Betracht ziehen, dass es sich bei diesen Texten um keine Fachwörterbücher handelt, d. h., sie müssen zugleich interessant für den/die Rezipienten/Rezipientinnen bleiben. Wie einige Strategien realisiert werden

71 Es wird nicht explizit angegeben, aber es lässt sich vermuten, dass die Wissenschaftler/Wissenschaftlerinnen an den Körper des untersuchten Tieres gerade dieses Halsband mit dem Sender befestigt haben.

72 Komplementäre bis dominante Beziehung (vgl. Stöck1 2004:250).

73 D. h. redundante Beziehung des Textes und des visuellen Begleitmaterials bei diesem konkreten Kommentar (nicht einmal wird dieses Halsband mit dem Sender detailliert gezeigt). Das Video mit den Tätigkeiten und der Lebensweise des Weibchens (Pflege der Jungtiere) ist sonst für die ganze Passage zu diesem Thema relevant. 
können, wird im Folgenden an zwei deutschen Beispielen präsentiert, wobei jeweils ein Beispiel aus der Fernsehsendung für Kinder und für Erwachsene stammt (siehe Tabelle 7).

\begin{tabular}{|c|c|}
\hline $\begin{array}{l}\text { K / D } \\
\text {,Lexikon“ }\end{array}$ & $\begin{array}{l}\text { Beispiel } 23 \\
\text { Rauchen. In einer Zigarette steckt Nikotin, eines der stärksten bekannten Gifte. [...] } \\
\text { Wenn sie die Zigaretten dann besser vertragen, beginnen Raucher eine schenbar positive } \\
\text { Wirkung zu spüren. [...] Dadurch entsteht direkt nach dem Inhalieren ein gutes Gefühl. } \\
\text { Der Körper muss das giftige Nikotin aber wieder loswerden - und baut das ab. Das } \\
\text { verursacht bei vielen Rauchern Unruhe oder Gereiztheit. Also - rauchen sie wieder. Sie } \\
\text { sind körperlich abhängig. Viele Raucher sind aber auch psychisch abhängig. Das heißt, } \\
\text { in bestimmten Situationen sehnen sie sich nach einer Zigarette. Sie haben so oft in diesen } \\
\text { Situationen geraucht, zum Beispiel in einer Kaffeepause, dass ihr Gehirn mittlerweile } \\
\text { darauf trainiert ist. Eine Pause ohne Zigarette ist dann einfach nicht mehr so schön. Diese } \\
\text { psychische Abhängigkeit wirkt besonders stark. [...] } \\
\text { URL } 24\end{array}$ \\
\hline $\begin{array}{l}\text { E / D } \\
\text {,Wilde Welt }\end{array}$ & $\begin{array}{l}\text { Beispiel } 24 \\
\text { [...] Mit rund } 360000 \text { Tieren kommen westliche Flachlandgorillas zwar am häufigsten } \\
\text { vor, doch auch sie sind vom Aussterben bedroht. Vom Cross-River-Gorilla sind maximal } \\
250 \text { Tiere übrig, er hat fast keine Chance mehr. Vom östlichen Flachlandgorilla gibt es } \\
\text { noch rund } 4000 \text { Tiere. Durch Verdrängung des Lebensraumes, Wilderei und Krankheiten } \\
\text { werden alle drei Unterarten immer weniger. Aufwärts geht es nur beim Berggorilla. } \\
\text { Knapp über } 1000 \text { Exemplare streichen in drei Ländern durch die Berge. [...] } \\
\text { URL } 15\end{array}$ \\
\hline
\end{tabular}

Tab. 7: Kombinierte Definitionen, Gliederung des Textes

Anmerkung: $\mathrm{K}=$ Fernsehsendung für Kinder, $\mathrm{E}=$ Fernsehsendung für Erwachsene, $\mathrm{D}=$ deutsche Fernsehsendung, $\mathrm{T}=$ tschechische Fernsehsendung

\section{Beispiel 23}

Das Beispiel 23 aus der Fernsehsendung ,Lexikon' wird bereits durch die Diskrepanz zwischen dem zu definierenden Fachwort/Lemma Rauchen und dem darauffolgenden Text spezifiziert. Man beginnt nämlich nicht damit, was Rauchen ist, sondern gleich mit der Zusammensetzung einer Zigarette. Dabei wird ein anderes Fachwort mithilfe einer Apposition definiert, und zwar das Nikotin als ein Gift. ${ }^{74}$ Man setzt hier wohl auf einen erzieherischen Effekt einer solchen Textgestaltung.

Der Definition von Nikotin folgen Definitionen von zwei terminologischen Wortverbindungen: körperlich abhängig und psychisch abhängig. Obwohl diese terminologischen Wortverbindungen verschiedene Kohyponyme des hyperonymischen Fachwortes „abhängig“ darstellen, werden sie nicht gleich definiert bzw. erklärt. Das Fachwort körperlich abhängig ist kataphorisch definiert und diese Definition bzw. Erklärung basiert auf einem Beispiel, d. h., es wird die Entwicklung der physischen Anhängigkeit schrittweise beschrieben, ${ }^{75}$ was dann in dem Fachwort körperlich abhängig zusammengefasst wird. Das Fachwort wird sprachlich nicht durch eine explizite Angabe „das ist“ oder „das nennt man“ eingeleitet, der kausale Zusammenhang lässt sich aber logisch ableiten und es wird auch durch die Kohäsion der Passage mithilfe des Topiks mit Wortbildungselementen unterstützt (Körper - körperlich). ${ }^{76}$

Bei dem Fachwort psychisch abhängig geht man umgekehrt vor, als man wegen der Systematisierung wohl erwartet hätte. Zuerst wird an das Fachwort physisch abhängig angeknüpft, erst

74 Es erinnert an die Definitionsart ,,taxonomisch-nominale Begriffserklärung“ bei Arntz/Picht/Schmitz (2014: 64-65) bzw. klassische, sog. aristotelische Definition (Roelcke 2010:62).

75 Es erinnert an die Definitionsart ,genetisch-kausale Bestimmung“ bei Arntz/Picht/Schmitz (2014:64-65) bzw. ,genetische oder operationale“ Definition bei Roelcke (2010:67).

76 Vgl. Wolf (2008:63). 
danach kommt die Charakteristik (anaphorische Definition). Diese Definitionen sind also spiegelbildlich gestaltet. Diese Passage ist kohärent aufgrund der Repetition gleicher Lexeme (abhängig), ${ }^{77}$ was einerseits die Textstruktur unterstützt. Diese Gliederung, die nicht nach einem einheitlichen Satzmuster gebildet wird, kann andererseits höhere Ansprüche an die Konzentration stellen, denn die Textgestaltung entspricht nicht der erwarteten Thema-Rhema-Struktur bzw. wechseln der Typ des Themas und des Rhemas. ${ }^{78}$ Am Ende wird die terminologische Wortverbindung noch einmal in einer lexikalisch modifizierten, d. h. substantivierten Form als Syntagma (gebildet aus Adjektiv und Substativ) aufgenommen, wodurch es zur Festigung der Kenntnis bei den Kindern kommen kann.

Ein anderes Spezifikum stellt dar, dass die Fachwörter psychisch abhängig und körperlich abhängig auf dem Bildschirm schriftlich gezeigt werden, ${ }^{79}$ während sie genannt werden, und zwar mit einer Schrift der alten Schreibmaschine bzw. mit Buchstaben, die weiße Ränder haben, als ob sie von einem Text ausgeschnitten und wieder eingeklebt wären. Es ist möglich, dass man sich diese doppelt angegebenen Fachwörter besser merkt und als relevant betrachtet, der Text schien aber dadurch nicht ganz beeinflusst zu sein. Die mündlich gesagten Erklärungen sind nämlich nicht reduziert und die Definition im Kommentar bei psychisch abhängig wird durch den Ausdruck ,das heißt" eingeleitet. Also haben diese schriftlich angegebenen Fachwörter den gesprochenen Text nicht ersetzt und können deswegen vielmehr als unterwertig bzw. redundant bezeichnet werden.

\section{Zusammenspiel des Verbalen und Bildlichen}

Redundant ist auch das sonstige begleitende visuelle Material, da es nur der Illustrierung und dem leichteren Merken dient, indem man bei Kindern Emotionen auslösen und ein bisschen Spaß machen will. Die Bilder sind unterschiedlichen Charakters. Am Anfang wird eine Animation des Zigarettenanzündens gezeigt. Dann folgen einzelne Fotos (d. h. statische Bilder) von einem Mann in verschiedenen Situationen, die sich durch Mimik und Gestik des Mannes unterscheiden: ${ }^{80}$ Wenn über negative Wirkungen des Nikotins gesprochen wird, schaut er finster drein, er hält seinen Bauch oder droht mit seinem Finger. Wenn über scheinbar positive Wirkung des Nikotins gesprochen wird, hält er eine Zigarette und lacht oder er macht die Geste mit dem gestreckten Daumen als Ausdruck von etwas Positivem.

Zusätzlich werden weitere Abbilder oder Animationen eingesetzt: ein Piktogramm (Totenschädel mit gekreuzten Knochen in einem Dreieck) an der Brust eines Menschen zur Andeutung der Giftigkeit des Nikotins, ein Bild eines Gehirns mit sich bewegenden hellen Wellenlinien (es erinnert an einen elektrischen Strom) zur Andeutung verschiedener Reaktionen aufgrund des Nikotininhalierens, ein Becher Kaffee zur Andeutung einer Pause, rote, vom Kopf des Mannes ausgehende und sich weiter um den Kopf herum vergrößernde Kreise ${ }^{81}$ zur Andeutung der Lust auf eine Zigarette und psychischer Abhängigkeit usw.

Es ist interessant, dass kein reales Video gezeigt wurde. Es kann vorausgesetzt werden, dass das visuelle Material nicht zu viel vom Gesprochenen ablenken sollte. Trotz des redundanten Charakters kann nicht gesagt werden, dass Bilder (oder Inschriften) völlig ausgelassen werden können, denn gerade die Aufmerksamkeit und Emotionen hervorrufenden Elemente spielen eine wichtige Rolle (vgl. Vaňková 2014), umso mehr, wenn es sich um ein gesellschaftlich relevantes Thema handelt.

\footnotetext{
Vgl. Wolf (2008:63).

78 Vgl. Problematik der Textverständlichkeit - Struktur (Göpferich 2001:130-132)

79 Dieser konzeptuelle bzw. graphische Unterschied von anderen analysierten Fernsehsendungen wurde neben dem ,Lexikon“ noch bei der tschechischen Sendung ,Kvítí verzeichnet (zum Konzept der Sendung siehe das Unterkapitel 4.1), in der die vorgestellten Pflanzen und deren tschechische (am Anfang der Sendung) und lateinische (am Ende der Sendung) Bezeichnungen samt einigen zusammenhängenden Fachwörtern auch schriftlich auf dem Bildschirm angegeben werden. Dieser Unterschied ist wohl durch den lexikonartigen Charakter beider Sendungen bedingt.

$80 \quad$ D. h. Abbilder (vgl. Weidenmann 1994:12-25) und Indexe (vgl. Burger/Luginbühl 2014:413-415) als Typen des Bildes.

${ }^{81}$ Vgl. schematisches Bild (Weidenmann 1994:12-25).
} 


\section{Beispiel 24}

Eine solche Gliederung des Textes mit Fachwörtern, deren Erklärungen oder Beschreibungen durch keinen gleichen Satzaufbau gebildet werden, kann man auch in einer der Fernsehsendungen für Erwachsene beobachten. Es handelt sich um die Vorstellung und Einführung mehrerer Unterarten von Gorillas, die weiter charakterisiert werden. ${ }^{82}$ Auch hier gilt, dass die Angabe der Unterartnamen (Gliederung der Gorillas) und die Beschreibung der Anzahl von Unterarten und ihre Lebensweise nicht ganz einheitlich sind. Die Informationen werden in verschiedener Reihenfolge gesagt, was eine erhöhte Konzentration erfordert, zugleich aber dem Text keine monotone Wirkung verleiht.

Im ersten Satz über westliche Flachlandgorillas wird zuerst die Anzahl der Exemplare angegeben und erst dann die Unterart beschrieben. Einen umgekehrten Aufbau haben dagegen Sätze, die Cross-River-Gorilla und östliche Flachlandgorilla vorstellen (zuerst Unterarten, dann Anzahl der Tiere). Eine kombinierte Reihenfolge der Information sieht man in der Passage zu Berggorillas. Hier erscheint zuerst die allgemeine Information darüber, dass die Anzahl steigt, dann folgt der Name der Unterart und erst im nächsten Satz kommt die konkrete Anzahl vor. Es ist wohl deswegen, dass man mithilfe der Satzgliedstellung (aufwärts in der betonten Position) besser auf den Kontrast zwischen aussterbenden und nicht aussterbenden Unterarten hindeuten kann, als wenn man nur die große Anzahl ohne Betonung angegeben hätte.

\section{Zusammenspiel des Verbalen und Bildlichen}

Zum Verstehen und guter Orientierung trägt das visuelle Begleitmaterial wesentlich bei. Immer wenn die konkrete Unterart und ihre Anzahl besprochen werden, wird eine Landkarte gezeigt, in der durch farbliche Fläche angedeutet ist, an welchen Orten sich diese Unterart befindet/(noch) lebt. ${ }^{83}$ Das Aussterben oder die Erweiterung der Unterart wird durch die Animation andeutet, indem sich die farbliche Fläche verringert oder vergrößert. Neben dieser Fläche in der Landkarte taucht immer noch ein rundes Bild mit dem Foto der Unterart auf, sodass man sich sowohl das entsprechende Tier als auch seinen sich verändernden Lebensraum sehr gut vorstellen und Informationen verbinden kann. Man ist also nicht nur auf den dabei gesprochenen Kommentar angewiesen, der auch in einer ausführlicheren Form die Informationen des visuellen Begleitmaterials nur schwierig umfassen könnte. ${ }^{84}$

\section{Auswertung}

Mit den neu eingeführten und zu definierenden/erklärenden Fachwörtern wird in analysierten Wissensvermittlungsfernsehsendungen für Kinder und für Erwachsene auf verschiedene Art und Weise gearbeitet.

Es wurden im Prinzip drei Situationen beobachtet. Erstens weisen bestimmte Phrasen oder entsprechende Prädikate im Satz auf das Fachwort oder die Definition hin. Zu diesen Verben gehören z. B. „nennen“, „,erkennen“, „heißen“, „,bedeuten“ usw. (bezogen auch auf die tschechischen Äquivalente), wobei diese Formulierungen entweder unpersönlich, z. B. mithilfe des Passivs, oder persönlich ausgedrückt werden, indem die benennenden Menschen spezifiziert werden oder sich die Rezipienten/Rezipientinnen selbst dadurch angesprochen fühlen können.

Zweitens werden Fachwörter oder Definitionen vom anderen Text nur durch eine Pause abgegrenzt, die die einleitenden und beschreibenden Verben ersetzt und einer gewissen Hervorhebung dient. Zum Beispiel wird auf diese Art und Weise an ein Fachwort, deren lexikalische Motivation nicht gleich durchschaubar ist, ein anderes Fachwort mit einer durchschaubaren Motivation angehängt oder eine Erklärung wird nach einer kurzen Pause in ein Fachwort kondensiert o. Ä.

Drittens wurden Definitionen verzeichnet, die im Text implizit erscheinen, und ihre Verbundenheit mit dem Fachwort kann aufgrund der Mittel der Kohäsion und Kohärenz abgeleitet werden.

\footnotetext{
82 Es erinnert an die Definitionsart „Klassifikation und Verweise“ bei Arntz/Picht/Schmitz (2014:64-65).

83 D. h. schematische Bilder (vgl. Weidenmann 1994:12-25).

84 Komplementäre bis dominante Beziehung (vgl. Stöckl 2004:250).
} 
Die analysierten Fachwörter werden durch kataphorische oder anaphorische Definitionen nach Niederhauser (1999) eingeführt. Sie stellen meistens keine offizielle, ausführliche wissenschaftliche Definition dar, sondern von den Denotaten werden nur einige relevante Attribute erwähnt oder durch Beispiele verdeutlicht. Einige Definitionen erinnern an Definitionsarten nach Arntz/Picht/Schmitz (2014) und Roelcke (2010). In einigen Erklärungen wird die Ursache der Benennung explizit gemacht.

Allgemein lässt sich also zusammenfassen, dass die Definitionen und Erklärungen - auch trotz einleitender Phrasen - vielmehr unauffällig vorkommen. Es wird angestrebt, sie im sonstigen Text zu „verbergen“, indem der umgebende Text abwechslungsreich gestaltet wird und verschiedene Typen der Definitionen verwendet werden (nicht nur „klassische“, sog. aristotelische Definitionen). In einigen Fällen werden umgangssprachliche Ausdrücke oder Metaphern und weitere Stilfiguren sowie abweichende Satzkonstruktionen verwendet, um die Kommentare den Rezipienten/Rezipientinnen aufzufrischen und zugänglicher zu machen.

Die gleiche Strategie wurde bei analysierten Gliederungen beobachtet. Der Text soll nicht zu wissenschaftlich wirken, sodass man die Fachwörter in einer Gruppe oder Hierarchie nicht nach einem einheitlichen Muster vorstellt, sondern verschiedene Reihenfolgen der Informationen, Kombinationen der kataphorischen und anaphorischen Definitionen usw. verwendet.

Die analysierten Definitionen/Erklärungen werden durch die nonverbalen Elemente (vor allem des Typs Abbild bzw. Ikon) unterstützt. Das visuelle Begleitmaterial übt verschiedene Funktionen aus. Einerseits wird auf relevante Aspekte in Videos aus der realen Natur deiktisch hingewiesen, sodass man das Aussehen der Objekte direkt zur Verfügung hat und ein nicht so umfangreicher sprachlicher Aufwand zur Vermittlung dieser Kenntnis investiert werden muss. Bei einigen komplizierteren Prozessen (z. B. Bestäubung) kann die Animation das richtige Verständnis der einzelnen Schritte der Prozesse vereinfachen. Andererseits sind einige Animationen, Bilder oder Videos in analysierten Fernsehsendungen zum Verstehen nicht unbedingt notwendig, sie sollen die Zuschauer/ Zuschauerinnen vielmehr beeindrucken oder unterhalten. Die Beziehungen zwischen den sprachlichen und bildlichen Darstellungen sind also meistens komplementär oder redundant.

Es konnten keine großen qualitativen Unterschiede in dem Sprachstil der Präsentation von Fachwörtern zwischen deutschen und tschechischen Fernsehsendungen für Kinder verzeichnet werden. Die festgestellten Strategien bei den Kinderfernsehsendungen treffen in großem Maße auch auf die analysierten Fernsehsendungen für Erwachsene zu. In einigen verglichenen Textpassagen wurden paradoxerweise sogar Sprachmittel beobachtet, die man vielmehr bei Kindersendungen erwarten würde und umgekehrt (z. B. Passiv bei den Kindersendungen gegen persönliche Formulierung bei den Fernsehsendungen für Erwachsene). Weiterhin hat sich bei einigen Kinderfernsehsendungen erwiesen, dass es schwierig ist, die Sprache und Inhalt den Kindern anzupassen und dabei ganz genau zu bleiben. In einem Fall wurde eine winzige Revision des Kommentars empfohlen.

Die kleineren präsentierten Differenzen zwischen einzelnen verglichenen parallelen Passagen sind problematisch zu verallgemeinern, denn sie sind durch die Auswahl der Beispiele bedingt oder können auf die unterschiedlichen biologischen Unterthemen zurückgeführt werden. Einige können mit dem unterschiedlichen Charakter der deutschen und tschechischen Sprache verbunden werden. Es lässt sich des Weiteren voraussetzen, dass der Individualstil der Autoren/Autorinnen die einzelnen Fernsehsendungen prägt. ${ }^{85}$

Trotzdem kann nicht geschlussfolgert werden, dass die Fernsehsendungen für verschiedene Altersgruppen überwiegend gleich sind. Es gibt Hinweise auf Unterschiede, die aus einer etwas unterschiedlichen Konzeption der jeweiligen Fernsehsendungen folgen. Diese Differenzen sind vor allem struktureller und inhaltlicher Art (Zeitproportionen, Organisation der Sendung, Menge der auftretenden Wissenschaftler/Wissenschaftlerinnen, positive/negative Wirkung der Nachrichten usw.). Eine spezifische Rolle kann den paraverbalen Mitteln zugeschrieben werden (Stimme des Kommentators, Modulationen der Intonation usw.).

85 Zum Individualstil vgl. Göttert/Jungen (2004:21-23). 
Entscheidend für die Feststellung der sprachlichen Unterschiede sind immer die Kombination und die synergetische Wirkung mehrerer Merkmale und Stilmittel, sodass sie bei jeder Textpassage einzeln beurteilt werden müssten. Es lassen sich Indizien verfolgen, dass der Vergleich anders ausgegangen wäre, wenn andere Aspekte der Sprache konfrontiert worden wären als die Arbeit mit den Fachwörtern (z. B. Arbeit mit Stereotypen, Maßangaben, erzieherischen Elementen usw.).

\section{Fazit und Ausblick}

Das Ziel des Artikels war herauszufinden, durch welchen Sprachstil es zur Wissensvermittlung in Wissensvermittlungsfernsehsendungen für Kinder kommt und ob darin Unterschiede zwischen deutschen und tschechischen Fernsehsendungen bestehen. Dieser Grundfrage wurde am Beispiel von vier ausgewählten Kinderfernsehsendungen zur biologischen Problematik nachgegangen. Vor allem konzentrierte sich die Untersuchung darauf, wie die Fachwörter eingeführt und erklärt/ definiert werden. Neben dem Sprachstil wurden nonverbale und paraverbale Aspekte bei der Analyse berücksichtigt. Um Spezifika der Kinderfernsehsendungen feststellen zu können, wurden zwei Fernsehsendungen für Erwachsene über Natur in die Analyse einbezogen. Es wurde eine qualitative Analyse der ausgewählten Fernsehserien durchgeführt und ausgewählte Passagen jeweils mit mindestens einem vergleichbaren Merkmal gegenübergestellt.

Die qualitative Analyse belegte keine großen Unterschiede sowohl zwischen deutschen und tschechischen Fernsehsendungen für Kinder als auch zwischen Fernsehsendungen für Kinder und für Erwachsene. In mehreren analysierten Fernsehsendungen findet man Parallelen in Textpassagen, die wahrscheinlich dadurch gegeben sind, dass man ein ähnliches Ziel verfolgt, die Liebe zur Natur und Erstaunen bei den Zuschauern/Zuschauerinnen hervorzurufen und zur Bewunderung der Natur zu motivieren. In analysierten Sendungen werden ähnliche Strategien und Methoden zum Aufmerksamkeit-Erwecken verwendet und entsprechende Sprachmittel ausgewählt, z. B. Metaphern, umgangssprachliche Wörter, Stilfiguren und Abweichungen von den Satzkonstruktionen. Auf die Fachwörter und Definitionen wird mithilfe verschiedener einleitender verbaler oder paraverbaler Mittel hingewiesen, einige kommen dagegen im Kommentar ohne Hinweis vor. Die Typen der Definitionen wechseln im Text oder sind kombiniert, da die Attraktivität des Kommentars der strengen Systematik vorgezogen wird.

Die Fachwörter werden nur in einem notwendigen Umfang erklärt, auf Details wird verzichtet. Der Erklärung von einigen Fachwörtern hilft das begleitende visuelle Material, in einigen Fällen funktioniert es jedoch mehr als Unterstützung des Interesses oder des Merkens. Die kleinen Unterschiede zwischen analysierten Fernsehsendungen sind teilweise durch deren unterschiedliche Konzepte bedingt und hängen mit der individuellen Auswahl von konkreten Sprachmitteln jeweils bei verglichenen äquivalenten Definitionen/Erklärungen zusammen.

Es muss hervorgehoben werden, dass diese Ergebnisse punktuell sind, sich nur auf ausgewählte Aspekte beziehen und deswegen eine begrenzte Gültigkeit haben. Für die objektiveren Ergebnisse müssten in die Analyse mehr Wissensvermittlungsfernsehsendungen einbezogen werden und zusätzlich eine Inhaltsanalyse und eine quantitative Analyse durchgeführt werden, die die Relevanz der einzelnen beobachteten Stilmittel nach ihrer Frequenz und dadurch ihre Relevanz bei der Konfrontierung der Fernsehsendungen determinieren können.

Damit eine komplexe Übersicht über die Wissensvermittlung in Fernsehsendungen gewonnen werden kann, sollten sich nächste Untersuchungen darüber hinaus der Perspektive der Rezipienten/ Rezipientinnen - deren Verständnis, Bedürfnissen und Stellungnahmen beim Rezeptionsprozess tiefer widmen. 


\section{Literaturverzeichnis}

\section{Primärliteratur:}

URL 3: ARD, WDR, ZDF. Neuneinhalb. Deine Reporter. Lexikon-Video. https://kinder.wdr.de/tv/ neuneinhalb/mehrwissen/lexikon-videos100.html [07.06.2021].

URL 4: ARD, ZDF. Southwell, Ben et al. KiKA. Tierbabys mit der Ente. https://www.kika.de/tierbabys-mit-der-ente/buendelgruppe2728.html [07.06.2021].

URL 5: ČT. Procházková, Maria et al. Déčko. Živý svět: Kvití. https://decko.ceskatelevize.cz/zivysvet-kviti [07.06.2021].

URL 6: ČT. Fulínová, Markéta/Polák, Marián et al. Déčko. Ptačí rodinky. To nejzajímavějši z ptačího světa. https://decko.ceskatelevize.cz/ptaci-rodinky [07.06.2021].

URL 12: ZDF. Gesang, Iris/Kieling, Andreas et al. Kielings wilde Welt. (Terra X). https://www. $\mathrm{zdf}$.de/suche?q=\%22Kielings + wilde + Welt $\% 22 \&$ synth $=$ true\&sender $=$ Gesamtes + Angebot $\&$ from $=\&$ to $=\&$ attrs $=\& a b N a m e=a b-2020-11-23 \&$ abGroup $=$ gruppe-d [14.06.2021].

URL 13:ZDF. Gesang, Iris/Kieling, Andreas et al. Kielings wilde Welt. Wildnis in Gefahr. https://www. zdf.de/dokumentation/terra-x/kielings-wilde-welt-wildnis-in-gefahr-100.html [14.06.2021].

URL 14: ZDF. Gesang, Iris/Kieling, Andreas et al. Kielings wilde Welt. Die Überlebenskünstler. https://www.zdf.de/dokumentation/terra-x/kielings-wilde-welt-die-ueberlebenskuenstler-100. html_[14.06.2021].

URL 15: ZDF. Gesang, Iris/Kieling, Andreas et al. Kielings wilde Welt. Geschützte Welten. https://www.zdf.de/dokumentation/terra-x/kielings-wilde-welt-geschuetzte-welten-100.html [14.06.2021].

URL 16: ČT: Thomas, Rosie/Badger, Giles. My, chytři primáti. https://www.ceskatelevize.cz/porady/11037889894-my-chytri-primati/ [14.06.2021].

URL 17: ČT: Thomas, Rosie/Badger, Giles. My, chytři primáti. Setkánís rodinou (Serienteil 1). https:// www.ceskatelevize.cz/porady/11037889894-my-chytri-primati/215382559960001-setkani-srodinou/ [14.06.2021].

URL 18: ČT: Thomas, Rosie/Badger, Giles. My, chytř́ primáti. Důležitost rodiny. (Serienteil 2) https://www.ceskatelevize.cz/porady/11037889894-my-chytri-primati/215382559960002-dulezitost-rodiny/ [14.06.2021].

URL 19: ČT: Thomas, Rosie/Badger, Giles. My, chytři primáti. Dar inteligence. (Serienteil 3) https://www.ceskatelevize.cz/porady/11037889894-my-chytri-primati/215382559960003-darinteligence/ [14.06.2021].

URL 20: ARD, WDR, ZDF. Neuneinhalb. Deine Reporter. Lexikon-Video. Bienen. https://kinder. wdr.de/tv/neuneinhalb/mehrwissen/lexikon/lexikon-video102.html [14.06.2021].

URL 21: ARD, ZDF. Southwell, Ben et al. KiKA. Tierbabys mit der Ente. Sprechen. https://www. kika.de/tierbabys-mit-der-ente/sendungen/sprechen-100.html [08.08.2020].

URL 22: ARD, ZDF. Southwell, Ben et al. KiKA. Tierbabys mit der Ente. Zu heiß, zu kalt. https:// www.kika.de/tierbabys-mit-der-ente/sendungen/sendung114478.html [08.08.2020].

URL 23: ARD, ZDF. Southwell, Ben et al. KiKA. Tierbabys mit der Ente. Schlafen. https://www. kika.de/tierbabys-mit-der-ente/sendungen/sendung114492.html [08.08.2020].

URL 24: ARD, WDR, ZDF. Neuneinhalb. Deine Reporter. Lexikon-Video. Rauchen. https://kinder. wdr.de/tv/neuneinhalb/mehrwissen/lexikon/lexikon-video132.html [14.06.2021].

URL 25: ARD, WDR, ZDF. Neuneinhalb. Deine Reporter. Lexikon-Video. Koffein. https://kinder. wdr.de/tv/neuneinhalb/mehrwissen/lexikon/lexikon-video-koffein100.html [07.06.2021].

URL 27: ARD, WDR, ZDF. Neuneinhalb. Deine Reporter. Lexikon-Video. Moor. https://kinder.wdr. $\mathrm{de} / \mathrm{tv} /$ neuneinhalb/mehrwissen/lexikon/lexikon-video118.html [07.06.2021].

\section{Sekundärliteratur:}

ARnTZ, Reiner / Picht, Heribert / Schmitz, Klaus-Dirk (2014): Einführung in die Terminologiearbeit. Hildesheim. 
Berk, Laura E. (2020): Entwicklungspsychologie. Hallbergmoos.

Breindl, Eva / Wassner, Ulrich H. (2006): Syndese vs. Asyndese. In: BlüHdorn, Hardarik/BreIndL, Eva/Wassner, Ulrich H. (Hrsg.): Text-Verstehen. Grammatik und darüber hinaus. Berlin; New York, S. 46-70.

BuCHER, Hans Jürgen (2017): Multimodalität als Herausforderung für die Visuelle Kommunikationsforschung. Wiesbaden. Zugänglich unter: https://www.researchgate.net/publication/317014015 Multimodalitat_als_Herausforderung_fur_die_Visuelle_Kommunikationsforschung; DOI: 10.1007/978-3-658-06738-0_31-2_[14.06.2020].

Burger, Harald / LuginBÜHL, Martin (2014): Mediensprache: eine Einführung in Sprache und Kommunikationsformen der Massenmedien. Berlin.

ČmeJRKovÁ, Světla / Daneš, František / SvĚrLÁ, Jindra (1999): Jak napsat odborný text. Praha.

Eroms, Hans-Werner (2008): Stil und Stilistik. Eine Einführung. Berlin.

FäsSLER, Peter E. (1998): Die neuere Fachsprache der Biologie seit der Mitte des 19. Jahrhunderts unter besonderer Berücksichtigung der Genetik. In: Hoffmann, Lothar / KalverkämPer, Hartwig / Wiegand, Herbert Ernst (Hrsg.): Fachsprachen/Languages for Special Purposes. Halbband 1. Berlin u. a., S. $1260-1269$.

FrAAs, Claudia (1998): Lexikalisch-semantische Eigenschaften von Fachsprachen. In: Hoffmann, Lothar / KalverkämPer, Hartwig / Wiegand, Herbert Ernst (Hrsg.): Fachsprachen/Languages for Special Purposes. Halbband 1. Berlin u. a., S. 428-438.

GÖPFERICH, Susanne (2001): Von Hamburg nach Karlsruhe: Ein kommunikationsorientierter Bezugsrahmen zur Bewertung der Verständlichkeit von Texten. In: Fachsprache/International Journal of LSP, Jg. 23, Nr. 3-4, Wien, S. 117-138.

GöTteRT, Karl-Heinz / JungEn, Oliver (2004): Einführung in die Stilistik. München.

Hahn, Walther von (1983): Fachkommunikation: Entwicklung - Linguistische Konzepte - Betriebliche Beispiele. Berlin.

Haller, Berthold / Probst, Wilfried (1989): Botanische Exkursionen, Bd. II: Sommerhalbjahr. Berlin; Heidelberg. Zugänglich unter: https://link.springer.com/chapter/10.1007\% 2F978-3-662-48688-7_ 7 [17.06.2021].

HofFmanN, Lothar (1998): Syntaktische und morphologische Eigenschaften von Fachsprachen. In: Hoffmann, Lothar / Kalverkämper, Hartwig / Wiegand, Herbert Ernst (Hrsg.): Fachsprachen/ Languages for Special Purposes. Halbband 1. Berlin u. a., S. 416-427.

KozubíkovÁ ŠAndovÁ, Jana (2019): Proměny českého akademického diskurzu během posledních padesáti let. In: Časopis pro moderni filologii, Jg. 101, Nr. 1, Praha, S. 54-71. Zugänglich unter: https:/casopispromodernifilologii.ff.cuni.cz/wp-content/uploads/sites/9/2019/06/Jana_Kozubikova_Sandova_54-71.pdf [17.06.2021].

LAnger, Inghard / Schulz von Thun, Friedemann / TAusch, Reinhard (1974): Verständlichkeit in Schule, Verwaltung, Politik und Wissenschaft mit einem Selbsttrainingsprogramm zur verständlichen Gestaltung von Lehr- und Informationstexten. München; Basel.

LÖFFLER, Heinrich (2010): Germanistische Soziolinguistik. Berlin.

MaLÁ, Jiřina (2009): Stilistische Textanalyse. Grundlagen und Methoden. Brno.

NiEDERHAUSER, Jürg (1999): Wissenschaftliche und populärwissenschaftliche Vermittlung. Tübingen.

RoElcke, Thorsten (2010): Fachsprachen. Berlin.

RÖMER, Christine (2019): Der deutsche Wortschatz: Struktur, Regeln und Merkmale. Tübingen.

Schwitalla, Johannes (2012): Gesprochenes Deutsch. Eine Einführung. Berlin.

Siegler, Robert et al. (2016): Entwicklungspsychologie im Kindes- und Jugendalter. Berlin; Heidelberg. (eBook)

Siever, Christina Margrit (2015): Multimodale Kommunikation im Social Web Forschungsansätze und Analysen zu Text-Bild-Relationen. Frankfurt am Main. Zugänglich unter: https://www. researchgate.net/publication/287491450_Multimodale_Kommunikation_im_Social_Web_Forschungsansatze_und_Analysen_zu_Text-Bild-Relationen [14.06.2021].

STÖскL, Hartmut (2004): Die Sprache im Bild-Das Bild in der Sprache. Berlin. 
Tworek, Artur (2019): Phonologie / Phonetik / Phonodidaktik - zu den Prinzipien der Ausspracheschulung aus philologischer Sicht. In: Linguistische Treffen in Wrocław, Jg. 16 (II), Wrocław, S. 353-363. Zugänglich unter: https://doi.org/10.23817/lingtreff.16-27 [14.06.2021].

VAŇKOVÁ, Lenka (2014): Einleitend: Emotionalität im Text. In: VAŇKOVÁ, Lenka (Hrsg.): Emotionalität im Text. Tübingen, S. 11-18.

Weidenmann, Bernd (1994): Informierende Bilder. In: Weidenmann, Bernd (Hrsg.): Wissenserwerb mit Bildern. Instruktionale Bilder in Printmedien, Film/Video und Computerprogrammen. Bern u. a., S. 9-58.

Wolf, Norbert Richard (2008): Textsyntax und/oder Textstilistik. In: Fritz, Thomas A. / Koch, Günter / TRоsт, Igor (Hrsg.): Literaturstil-sprachwissenschaftlich. Heidelberg, S. 57-69.

\section{Internetquellen:}

URL 1: ARD, ZDF. KiKA. https://www.kika.de/index.html [07.06.2021].

URL 2: ČT. Déćko. https://decko.ceskatelevize.cz/ [07.06.2021].

URL 7: ARD, WDR, ZDF. Neuneinhalb - das Reportermagazin für Kinder. https://www.ardmediathek.de/daserste/sendung/neuneinhalb-das-reportermagazin-fuer-kinder/Y3JpZDovL2Rhc2Vyc3RlLmRIL25ldW5laW5oYWxi/ [07.06.2021].

URL 8: Česko-Slovenská filmová databáze. https://www.csfd.cz/film/715481-ptaci-rodinky/galerie/?type=1 [07.06.2021].

URL 9: Krejzová, Lenka et al. Mistní kultura. Aktuálni kulturni zpravodajství z domova; Autor článku: jal. Tři roky animace na Déčku. Česká televize představí na Anifilmu i nový večerníček https://mistnikultura.cz/tri-roky-animace-na-decku-ceska-televize-predstavi-na-anifilmu-i-novy-vecernicek [07.06.2021].

URL 10: Procházková, Maria. Zábavně-vzdělávací cykly pro děti. https://www.czechschoolsamerica.org/uploads/5/3/0/5/53056687/6.1_maria_prochazkova_vzdelavaci_porady_pro_deti.docx [07.06.2021].

URL 11: BBC. Andy's Baby Animals. https://www.bbc.co.uk/programmes/b083gr3k [07.06.2021].

URL 26: Kollektiv der Autoren: Duden - Onlinewörterbuch. Stichwort „Nektar“. https://www. duden.de/rechtschreibung/Nektar [07.06.2021].

URL 28: Kollektiv der Autoren: Duden - Onlinewörterbuch. Stichwort „,verlanden “. https://www. duden.de/rechtschreibung/verlanden [07.06.2021].

URL 29: ČT. Večerníček. https://www.ceskatelevize.cz/tv-program/hledani/?filtr[nadtitul] $=\mathrm{Ve} \% \mathrm{C} 4$ \%8Dern\%C3\%AD\%C4\%8Dek [16.06.2021].

URL 30: CBeebies (BBC). Andy's Baby Animals. Baby Lemurs catch a ride! https://www.youtube. com/watch?v=ZEiJnKDc5IU [17.06.2021]. 